\title{
An $\boldsymbol{R}_{\mathbf{0}}$ theory for source-sink dynamics with application to Dreissena competition
}

\author{
Martin Krkošek • Mark A. Lewis
}

Received: 23 May 2008 / Accepted: 29 March 2009/Published online: 28 May 2009

(C) Springer Science + Business Media B.V. 2009

\begin{abstract}
Source-sink dynamics may be ubiquitous in ecology. We developed a theory for source-sink dynamics using spatial extensions of the net reproductive value, $R_{0}$, which has been used elsewhere to define fitness, disease eradication, population growth, and invasion risk. $R_{0}$ decomposes into biologically meaningful componentslifetime reproductive output, survival, and dispersal-that are widely adaptable and easily interpreted. The theory provides a general quantitative means for relating fundamental niche, biotic interactions, dispersal, and species distributions. We applied the methods to Dreissena and found a resolution to a paradox in invasion biology - competitive coexistence between quagga (Dreissena bugensis) and zebra (D. polymorpha) mussels among lakes despite extensive niche overlap within lakes. Source-sink dynamics within lakes between deepwater and shallow habitats, which favor quagga and zebra mussels, respectively, yield a metacommunity distribution where quagga mussels dominate large lakes and zebra mussels dominate small lakes. The sourcesink framework may also be useful in spatial competition theory, habitat conservation, marine protected areas, and ecological responses to climate change.
\end{abstract}

M. Krkošek $(\bowtie) \cdot$ M. A. Lewis

Centre for Mathematical Biology,

Departments of Mathematical and Statistical Sciences

and Biological Sciences, University of Alberta,

Edmonton, AB T6E 2E7, Canada

e-mail: mkrkosek@u.washington.edu

M. A. Lewis

e-mail: mlewis@math.ualberta.ca

Present Address:

M. Krkošek

School of Aquatic and Fishery Sciences,

University of Washington,

Seattle, WA, USA
Keywords Competition · Dispersal · Survival . Source-sink dynamics $\cdot$ Niche theory $\cdot$ Dreissena

\section{Introduction}

Source-sink dynamics are central to ecology for their influence on population dynamics (Brown and KodricBrown 1977; Pulliam 1988), species ranges (MacArthur 1972; Holt 2003), and competitive coexistence (Amarasekare and Nisbet 2001; Snyder and Chesson 2004). Dispersal may be as important as competition in explaining differences between fundamental and realized niche (Hutchinson 1957; Pulliam 2000) because source-sink dynamics can maintain populations in poor habitat (Pulliam 1988) and extinguish them in suitable habitat (Amarasekare and Nisbet 2001). Understanding source-sink dynamics is also of applied importance for conserving species on fragmented landscapes (Hanski and Gyllenberg 1993; Hanski 1998) and designing marine protected areas (Lubchenco et al. 2003; Neubert 2003). Despite the fundamental and applied importance of source-sink dynamics, there is some confusion surrounding what actually constitutes a source or a sink and how to quantitatively distinguish between the two. The central confusion for population dynamics is whether sources should reflect the fundamental niche (Pulliam 2000) or the contribution a local population makes to the metapopulation (Figueira and Crowder 2006; Runge et al. 2006).

In this paper, we introduce a new theory for source-sink dynamics and apply it to a long-standing problem in invasion biology - competition between Dreissena mussels. The theory is based on the net reproductive value, $R_{0}$, which measures the number of progeny produced in the lifetime of a single individual (Heesterbeek 2002). We begin by briefly reviewing the theory of source-sink 
dynamics, with a focus on their relation to niche, dispersal, and distribution. We then develop the new theory for both single species and competing species cases, using a mutual invasibility analysis to assess scenarios of species persistence, exclusion, and coexistence. Our review and theoretical development brings together and quantifies concepts from competition theory, spatial ecology, and mathematical biology whose interrelatedness had not been fully appreciated. We follow by examining the Dreissenid paradox, which is the competitive coexistence of quagga (Dreissena bugensis) and zebra (D. polymorpha) mussels among lakes despite extensive niche overlap. Application of the theory to a spatial model of zebra and quagga mussel competition reveals source-sink dynamics between shallow and deepwater habitats that leads to within-lake exclusion and among-lake coexistence.

\section{Theory of source-sink dynamics}

Pulliam (1988) considered a population distributed over a fragmented landscape and suggested that population flow from source habitats can maintain populations in sink habitats - echoing the rescue effect proposed a decade earlier (Brown and Kodric-Brown 1977). Pulliam (1988) proposed two definitions for sources and sinks based on equilibrium and invasion (low density) conditions. The first definition considers a population at equilibrium (all subpopulation densities are constant through time) and defines a source as a net exporter and a sink as a net importer of individuals. The second definition considers a population at low density and defines a source as habitat patches in which a population can grow in the absence of immigration and emigration. The equilibrium definition has been used in the evolutionary theory of source-sink dynamics (Kawecki 1995) and the invasion definition has been used in population dynamics (Pulliam 1988), where it has recently been modified and redefined (Figueira and Crowder 2006; Runge et al. 2006). Our focus is on the invasion definition used in population dynamics, which allows us to be consistent with previous works and also allows us to use linear approximations that simplify the analysis.

In his original article, Pulliam (1988) used the local population growth rate (at low population size and no density dependence) of an annually breeding species,

$\lambda=P_{\mathrm{A}}+P_{\mathrm{J}} \beta$

to classify source and sink habitat patches. The parameter $P_{\mathrm{A}}$ is adult survival, $P_{\mathrm{J}}$ is juvenile survival, and $\beta$ is the number of juveniles produced per adult. All of $P_{\mathrm{A}}, P_{\mathrm{J}}$, and $\beta$ are habitat specific and so each habitat can have a different value of $\lambda$. Source patches were those with $\lambda>1$ and sinks were those with $\lambda<1$. Equation (1) is based on an annual breeding cycle but the local population growth rate is more generally expressed as the dominant eigenvalue of the linearized local population projection matrix for discrete-time stage or age-structured populations (Caswell 2001). Analogous classifications have been used in discussions of source-sink dynamics on patches (Fox 2007), and discretized (Pulliam 2000) and continuous (Snyder and Chesson 2004) landscapes.

Hutchinson (1957) introduced the idea of a species fundamental niche as a hypervolume defining the set of environmental conditions (e.g., moisture, salinity, $\mathrm{pH}$, irradiance, etc.) that can support the species within a space defined by environmental (not spatial) axes. According to source-sink theory based on local population growth rates, source habitat is therefore the projection of a fundamental niche onto geographical space. However, this can be misleading from the population perspective (Figueira and Crowder 2006; Runge et al. 2006). For example, Runge et al. (2006) described how a habitat could be classified as a source according to $\lambda$ and yet contribute no individuals to the population because of $100 \%$ mortality during emigration. Thus, a theory of source-sink dynamics needs to depart some from the niche concept and incorporate dispersal.

At least two studies have attempted to incorporate dispersal into the classification of source and sink habitat patches (Figueira and Crowder 2006; Runge et al. 2006). Runge et al. (2006) first proposed this and introduced a new criterion, $C^{r}$, for distinguishing source and sink habitats. $C^{r}$ represents the contribution of a focal population to the metapopulation by defining

$C^{r}=\phi_{\mathrm{A}}^{r r}+\sum_{s \neq r} \phi_{\mathrm{A}}^{r s}+\beta^{r}\left(\phi_{\mathrm{J}}^{r r}+\sum_{s \neq r} \phi_{\mathrm{J}}^{r s}\right)$

where $\phi_{\mathrm{A}}^{r s}$ and $\phi_{\mathrm{J}}^{r s}$ are the probabilities that an adult and juvenile, respectively, from patch $r$ survive to be in patch $s$ in the following year (Runge et al. 2006). Equation (2) is based on the same annual breeding cycle as Eq. (1) but now includes emigration of adults and juveniles from a focal patch to the metapopulation. That is, in one time step, an adult individual in patch $r$ will contribute $C^{r}$ individuals to the metapopulation. Figueira and Crowder (2006) made nearly the same development, defining a "contribution growth rate", $\lambda_{c}$, and applied it to mayflies and reef fish. Runge et al. (2006) went a step further by generalizing $C^{r}$ to stage- or age-structured populations although the calculations can be complex and the expressions may not be biologically intuitive (see appendix A in Runge et al. 2006).

Source-sink dynamics also arise in spatial competition models, most often as a mechanism underlying competitive coexistence (Amarasekare 2003; Snyder and Chesson 2004). They also appear as a process that can alter 
outcomes of competition such as species extirpation from what is otherwise suitable habitat (Amarasekare and Nisbet 2001; Schreiber and Kelton 2005). Many of these papers (e.g., Snyder and Chesson 2004; Schreiber and Kelton 2005) use the traditional definition of source or sink based on local growth rates similar to Pulliam (1988), and so suffer from the same limitations identified by Runge et al (2006) in that they may not reflect the contribution a local population makes to the metapopulation. There is large variation in the structure of competition models in which source-sink dynamics arise, ranging from continuous time models on patches (Amarasekare and Nisbet 2001) to discrete time models on continuous landscapes (Snyder and Chesson 2004). As such, there is no general mathematical framework for source-sink dynamics to study how distributions of sources and sinks are affected by competition and dispersal as well as how source-sink dynamics mediate the relations among niche, competition, and distribution.

A classification of source and sink habitats should accommodate dispersal to reflect the flow of individuals from a focal subpopulation to the larger population (Figueira and Crowder 2006; Runge et al. 2006). Such a theory should also be general in its application by extending beyond classical metapopulation formulations for single species with annual lifecycles. It should accommodate age- or stage-structured lifecycles as well as fragmented and continuous landscapes within a framework that is biologically intuitive. Further, it should also address classical problems such as relating niche and distribution as well as explaining competitive coexistence. We propose that such a theory exists when source-sink dynamics are viewed through the lens of the net reproductive value, $R_{0}$. $R_{0}$ theory offers the advantage of integrating demographic processes over an entire lifecycle, and because of its long history in demography and epidemiology (Heesterbeek 2002), it offers a well-developed quantitative toolkit for treating structured populations. We begin by briefly reviewing $R_{0}$ and then describe its application to source-sink dynamics in single species models, competitive coexistence, and the relationship between niche and distribution.

An $R_{0}$ theory for source-sink dynamics

A new perspective on source-sink dynamics comes via the net reproductive value, $R_{0}$, defined as the number of adult progeny produced in the lifetime of a single adult (Heesterbeek 2002). $R_{0}$ is prevalent in epidemiology, where it differentiates disease persistence and eradication (Diekmann et al. 1990; Anderson and May 1991; Diekmann and Heesterbeek 2000). It also appears in life history theory (Metz et al. 1992; Mylius and Diekmann 1995), age- or stage-structured population dynamics (Caswell 2001), and invasion biology (de Camino-Beck and Lewis 2007). In all cases, if $R_{0}>1$ a small population will establish/persist but if $R_{0}<1$ then individuals cannot on average replace themselves and the population declines to extinction (Cushing and Zhou 1994; $\mathrm{Li}$ and Schneider 2002). Note that $R_{0}$ theory is different and more general than $R^{*}$ theory, which predicts competitive exclusion based on minimal resource requirements (Tilman 1982), but which breaks down under dispersal (Abrams and Wilson 2004) and is limited to models of resource competition.

We use three spatial extensions of $R_{0}$ as the basis of source-sink theory. The first extension is a fundamental niche $R_{0}$, denoted $\widehat{R}_{0}(x)$, that determines the spatial distribution of a species fundamental niche $(x$ denotes space). The second is a source-sink $R_{0}$, denoted $R_{0}(x)$, that maps source and sink regions by accommodating dispersal. The third is a global $R_{0}$, denoted $\bar{R}_{0}$, that determines global species persistence and/or coexistence. Our theoretical development falls broadly within the existing mathematical framework for calculating $R_{0}$ for structured epidemics (Diekmann et al. 1990). We begin by considering models with single dispersal events in the lifecycle-i.e., populations with dispersing young and sessile adults (appropriate for many plants, algae, invertebrates, and some vertebrates) for which an $R_{0}$ theory for source-sink dynamics is most helpful. We provide the criteria for defining source and sink habitats in both patchy and continuous landscapes and illustrate how source-sink dynamics relate niche and distribution using generalized quantities-reproduction and survival - that can be extracted from a variety of population models.

\section{$R_{0}$ for single species models}

For populations with dispersing young and sessile adults, there are two spatially separated demographic processeslifetime reproductive output of progeny by an average reproducing adult (denoted by $\Lambda(x)$ ) and the probability progeny survive to become reproducing adults (survival, denoted by $\Psi(x))$. The first spatial extension of the net reproductive value, the niche $R_{0}$ denoted by $\widehat{R}_{0}(x)$, defines fundamental niche space and so, by definition, it strictly excludes dispersal and competition. It is simply

$$
\widehat{R}_{0}(x)=\underbrace{\Lambda(x)}_{\begin{array}{c}
\text { reproduction } \\
\text { at location } x
\end{array}} \cdot \underbrace{\Psi(x)}_{\begin{array}{c}
\text { survival } \\
\text { at location } x
\end{array}},
$$

which accounts for spatial variation in the abiotic environment. Suitable habitat consists of areas where the environment allows persistence $\left(\widehat{R}_{0}(x)>1\right)$. Locations where the population cannot establish $\left(\widehat{R}_{0}(x)<1\right)$ correspond to unsuitable habitat and are outside the fundamental niche.

To see the effects of dispersal on source-sink dynamics, we introduce a dispersal kernel, $k(x, z)$, which defines the 
probability density that a propagule released from location $x$ will settle at location $z$. The net reproductive value of an average individual at location $x$ must account for the dispersal from $x$ to $z$ and subsequent survival of offspring to adulthood at $z$ throughout a spatially continuous landscape

$$
R_{0}(x)=\underbrace{\Lambda(x)}_{\begin{array}{c}
\text { reproduction } \\
\text { at location } x
\end{array}} \underbrace{\int \Psi(z) k(x, z) d z}_{\begin{array}{c}
\text { survival } \\
\text { after dispersal from } x
\end{array}} .
$$

For the case where habitat is fragmented among discrete patches, we have

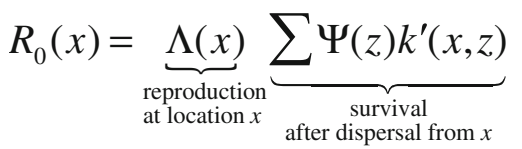

where $k^{\prime}(x, z)$ is the probability of moving from patch $x$ to patch $z$. For organisms with no mortality during dispersal, the dispersal kernel gives unity when summed over all possible settling locations $z$. If, in addition, the species becomes immediately reproductive, the survival term $\Psi(z)$ equals unity and so the sum in Eq. (5) also equals unity and the source or sink nature of patch $x$ is determined solely by local dynamics. Locations where $R_{0}(x)>1$ function as sources because individuals at location $x$ on average produce more than one adult offspring somewhere in the spatial domain. Locations where $R_{0}(x)<1$ function as sinks because on average the lifetime reproductive output of an individual at location $x$ results, on average, in less than one adult offspring.

Equations (4-5) map how source and sink distributions change with dispersal and abiotic factors. However, they do not inform on the global persistence or extinction of the focal species. To do this we need the concept of a next generation operator, $\Gamma$, which describes the expected density of population arising from a single individual whose precise location in space may be unknown, but is distributed by $\phi_{0}$. Any small population distributed by $\phi_{0}$ will be distributed in the next generation according to

$$
\Gamma \phi_{0}(x)=\underbrace{\Psi(x)}_{\begin{array}{c}
\text { survival } \\
\text { at location } x
\end{array}} \underbrace{\int \Lambda(z) \phi_{0}(z) k(z, x) d z}_{\begin{array}{c}
\text { density of propagules } \\
\text { arriving at } x
\end{array}} .
$$

For the case where habitat is fragmented among discrete patches, we have

$$
\Gamma \phi_{0}(x)=\underbrace{\Psi(x)}_{\begin{array}{c}
\text { survival } \\
\text { at location } x
\end{array}} \underbrace{\sum \Lambda(z) \phi_{0}(z) k^{\prime}(z, x)}_{\begin{array}{c}
\text { density of propagules } \\
\text { arriving at } x
\end{array}} .
$$

where $x$ and $z$ denote patch identities. That is, the next generation produced by a single individual distributed by $\phi_{0}(z)$ is determined by reproduction at all locations $z$, weighted by the proportion of the individual located there, $\phi_{0}(z)$, times the probability of dispersing from $z$ to $x, k(z, x)$, times the probability of being recruited at location $x$, and then summed over all possible starting locations $z$. Equations (6-7) lead to a calculation of the global net reproductive value, $\bar{R}_{0}$, that determines persistence. Note that Eqs. (6)-(7) are linear so the population will either persist or go extinct. This linear model can be posed as an eigenvalue-eigenvector problem to reveal $\bar{R}_{0}$ :

$\Gamma \phi(x)=\bar{R}_{0} \phi(x)$

The global net reproductive value is the dominant eigenvalue of the next generation operator, $\Gamma$, and the corresponding eigenvector is $\phi(x)$ (Diekmann et al. 1990). That is, after introduction, the population will grow at an intergenerational rate $\bar{R}_{0}$ and the spatial distribution of the invading population will stabilize at $\phi(x)$, providing reasonable assumptions on $\Lambda, \Psi$, and $k$ are met. The solutions for $\bar{R}_{0}$ and $\phi(x)$ can be found numerically via the power method (Press et al. 2002), which involves iterating and rescaling Eqs. (6)-(7) until the spatial distribution is stabilized. The distribution of the initial individual, $\phi_{0}(z)$, is arbitrary. The eigenfunction is the stabilized spatial distribution of individuals and the global net reproductive value (dominant eigenvalue) is the associated change in magnitude of population density between iterations. For this power method to work, the operator (6)-(7) must be superpositive, that is it must have a simple positive dominant eigenvalue with a positive eigenfunction, and no other eigenfunction is positive (Krasnosel'skii and Zabreiko 1984). This superpositivity is satisfied, for example, if $\Lambda$, $\Psi$, and $k$ are positive. The condition that $k$ is positive everywhere can be relaxed, providing individuals can disperse from each location to every other location in a finite number of steps (see Lutscher and Lewis 2004 for details). These conditions are usually met in ecological models featuring dispersal kernels, including the model of Dreissena competition that we analyze later in this paper.

In Appendix 1, we show how Eq. (8) can be used to recover mathematically and biologically pleasing solutions in two special cases: no dispersal and globally uniform dispersal.

Examples of single species models

The results from the preceding theory can be applied to previous models of spatial population dynamics. The application is particularly straightforward when the adults are sessile, and there is a single dispersal event associated with the settlement of new juveniles. We will focus on this case, deferring discussion of more complex life historydispersal patterns to the 'Discussion'. 
A single species with discrete non-overlapping generations and annual reproduction and dispersal events can be modeled with an integrodifference equation

$n(x, t+1)=\int f(n(z, t), z) n(z, t) k(x, z) \mathrm{d} z$

where $n(x, t)$ is the abundance of individuals at location $x$ and time $t$, and $f$ is a density-dependent reproduction function such as Ricker or Beverton-Holt population growth with nonnegative geometric growth rate $f(0, x)$. At low population densities, dynamics from one generation to the next are approximated by the linear equation

$n(x, t+1)=\int f(0, z) n(z, t) k(z, x) \mathrm{dz}=\Gamma \mathrm{n}(\mathrm{x}, \mathrm{t})$

For this model, the reproduction and survival terms for the $R_{0}$ theory are $\Lambda(x)=f(0, x)$ and $\Psi(x)=1$, and, with these, the next generation operator takes the form given in Eq. (6). If there is some density-dependent mortality of individuals following dispersal, with fraction $s(n, x)$ surviving, then Eq. (10) can be extended to

$n(x, t+1)=s(n(x, t), x) \int f(n(z, t), z) n(z, t) k(z, x) \mathrm{dz}$,

or

$$
n(x, t+1)=\int f(n(z, t), z) s(n(z, t), z) n(z, t) k(z, x) \mathrm{dz}
$$

depending whether mortality is before or after census. Both yield $\Lambda(x)=f(0, x)$ and $\Psi(x)=s(0, x)$.

How can this model be extended further to include overlapping generations? The simplest case, applicable to some plant and insect populations, involves dispersal of juveniles prior to a sedentary adult stage. This extends Eq. (9) to

$$
\begin{aligned}
n(x, t+1)= & s(n,(x, t), z) n(x, t) \\
& +\int f(n(z, t), z) s(n(z, t), z) n(z, t) k(z, x) \mathrm{dz}
\end{aligned}
$$

where the survival term $s$ is applied prior to reproduction. At low densities, dynamics from one year to the next are approximated by the linear equation

$$
\begin{aligned}
n(x, t+1)= & s(0, z) n(x, t) \\
& +\int f(0, z) s(0, z) n(z, t) k(z, x) \mathrm{dz} .
\end{aligned}
$$

Here, the lifespan of a reproducing adult is geometrically distributed with expectation $(1-s(0, x))^{-1}$ (see Appendix 3).
Thus, $\Lambda$ is the expected lifespan of an individual at location $x$ in the absence of density-dependent mortality times its annual reproductive output $\Lambda(x)=(1-s(0, x))^{-1} f(0, x)$ and $\Psi$ is the probability of newly produced juveniles surviving to adulthood $\Psi(x)=s(0, x)$. In this case, the next generation operator (6) takes the form

$$
\Gamma n(x, t)=s(0, x) \int(1-s(0, z))^{-1} f(0, z) n(z, t) k(z, x) \mathrm{d} z
$$

An extension of this model will be considered for the Driessenid competition dynamics in later sections of the paper.

A metapopulation example where $R_{0}$ theory may be preferable to $C^{r}$ is when the population is structured by stages and the number of patches is relatively large. Consider a lifecycle consisting of dispersing young, sessile juveniles, and sessile adults. The corresponding model is

$$
\begin{aligned}
n_{y}(x, t+1) & =\sum_{z} f(z) s_{a}(z) n_{a}(z, t) k^{\prime}(z, x) \\
n_{j}(x, t+1) & =s_{y}(x) n_{y}(x, t) \\
n_{a}(x, t+1) & =s_{j}(x) n_{j}(x, t)+s_{a}(x) n_{a}(x, t)
\end{aligned}
$$

where $n_{y}, n_{j}$, and $n_{a}$ are the three population stages-young, juveniles, and adults. The annual survival of each stage is $s_{y}, s_{j}$, and $s_{a}$, respectively. Only adults reproduce, producing $f(x)$ young each year. To apply $C^{r}$ theory, one must construct a transition matrix that connects the network of nodes representing every combination of population stage and patch location (Runge et al. 2006). The large number of possible combinations (in this case, three stages times the number of patches, squared) means the transition matrix can quickly become very large, encumbering calculations for the contribution metric or the metapopulation growth rate. Also, the homogenization of space and population stage into one transition matrix loses some biological appeal because the intuitive structuring of space and population stage is lost. However, application of $R_{0}$ theory is straightforward. The quantities of interest are lifetime reproductive output of young per adult $\Lambda(x)=f(x) \times$ $\left[1-s_{a}(x)\right]^{-1}$ and survival, the probability a young will survive to reach the adult stage, $\Psi(x)=s_{y}(x) s_{j}(x) s_{a}(x)$. If the network of patches was replaced with a continuous landscape of varying habitat quality, $C^{r}$ theory would not apply whereas $R_{0}$ theory remains straightforward.

$R_{0}$ for spatial competition models

Source-sink dynamics are one mechanism that can facilitate competitive coexistence (Amarasekare 2003). The $R_{0}$ framework can be extended to analyze spatial competition models and provide insights into the relations among niche, 
competition, dispersal, and distribution. To do so, we turn to a mutual invasibility analysis and consider multiple competing species each of which has its own non-zero equilibrium distribution in the absence of interspecific competition. The mutual invasibility analysis considers the net reproductive value of a focal species introduced at low abundance into a community of resident species at their equilibrium densities. In this situation, the focal species experiences competition with resident species, which may affect reproduction and/or survival. The invader is considered sufficiently rare to escape intra-specific competition. Below, we summarize how the principles of $R_{0}$ theory for source-sink dynamics can be applied to spatial competition models. We follow with a detailed application of the theory to Dreissena competition, which involves stage-structured integrodifference models.

The principles for the competing species case follow similarly from the single species case. We modify the notation replacing $\Lambda$ and $\Psi$ with $\Lambda^{i}$ and $\Psi^{i}$ to denote the effects of competition with resident species $r$ on reproduction and survival of the focal invading species $i$. Interspecific competition acts to reduce the values of $\Lambda^{i}$ and $\Psi^{i}$ relative to the single species case, but the exact effect is dependent on the nature of competition. Because the resident is at steady state, the values of $\Lambda^{i}$ and $\Psi^{i}$ are temporally constant but may be spatially variable. The effects of competition on distributions relative to fundamental niche can be seen by mathematically or graphically comparing $\widehat{R}_{0}(x)$ and $\widehat{R}_{0}^{i}(x)$. The effect of competition on source-sink dynamics can be viewed by comparing $R_{0}(x)$ and $R_{0}^{i}(x)$. The global net reproductive value defines if the focal invading species will grow (if $\bar{R}_{0}^{i}>1$ ) or decline if $\bar{R}_{0}^{i}<1$. Coexistence occurs when $\bar{R}_{0}^{i}>1$ for both species.

The behavior when one or both $\bar{R}_{0}^{i}<1$ depends upon the nature of the non-linear dynamics and requires more detailed analysis. General conditions for existence of a globally stable equilibrium value are that the dynamics are monotone (typical of two-species competitive systems), that the basin of attraction for the steady state for extinction of both species is trivial, that, in the absence of the other species, each species has a globally stable resident steady state (thus excluding possible Allee effects), and that at most one coexistence equilibrium exists (Smith and Theime 2001). Under these conditions, we expect competitive exclusion to occur if one species has $\bar{R}_{0}^{i}>1$ and the other has $\bar{R}_{0}^{i}<1$, and if both species have $\bar{R}_{0}^{i}<1$ then the dominant species is one that first becomes established (bistability). The more exotic case of semi-stability of the coexistence equilibrium is theoretically possible, but degenerate from a mathematical perspective, and thus unlikely to occur for biologically realistic models (see Discussion of Smith and Theime 2001). As described above, this relationship between $R_{0}$ and competitive outcomes implicitly relies upon the absence of Allee effects. If an Allee effect is present, there is the possibility of establishment when $\bar{R}_{0}^{i}<1$ but introduction must occur at sufficiently high levels to overcome depensation (Boldin 2006).

Summary of $R_{0}$ theory for source-sink dynamics

To summarize, we have shown how to map the boundaries of a fundamental niche by solving Eq. (3) - when there is no dispersal or competition-for $\widehat{R}_{0}(x)=1$. Areas where $\widehat{R}_{0}(x)>1$ are within the fundamental niche and correspond to positive equilibrium densities. Areas where $\widehat{R}_{0}(x)<1$ are outside the fundamental niche and cannot support a population. When dispersal is included, a fundamental niche may be exceeded through the rescue effect (immigration prevents local population extinction) (Brown and Kodric-Brown 1977). This occurs when the global net reproductive value is greater than unity, $\bar{R}_{0}>1$, because population growth occurs everywhere inside or outside the fundamental niche. The population growth can be sustained at sink locations $\left(R_{0}(x)<1\right)$ through immigration of propagules from source locations $\left(R_{0}(x)>1\right)$. Alternatively, the fundamental niche may not be filled because of strong dispersal coupling to extensive sink habitat. This occurs when the global net reproductive value is less than unity, $\bar{R}_{0}<1$; global population growth is negative everywhere, inside and outside the fundamental niche, and the population will eventually reach extinction. Regardless of whether the outcome is population persistence or extinction, one can map the spatial distributions of source and sink habitats as they function in response to the environment, competition, and dispersal. This is done by solving Eqs. (4)-(5) for $R_{0}(x)=$ 1. Regions where $R_{0}(x)>1$ function as sources and regions where $R_{0}(x)<1$ function as sinks. These regions characterize the source-sink dynamics underlying population persistence or extinction, but do not, in and of themselves, inform on whether the population will, as a whole, persist. Global persistence is only determined by the global net reproductive value, $\bar{R}_{0}$. The effects of competition on niche space, sourcesink dynamics, and global persistence can be seen by direct comparisons between $\widehat{R}_{0}(x)$ and $\widehat{R}_{0}^{i}(x), R_{0}(x)$ and $\bar{R}_{0}(x)$ and $\bar{R}_{0}^{i}(x)$. The actual example, illustrating how this can be done in practice, is given in the next sections that model competition between zebra and quagga mussels in lakes.

\section{Dreissenid competition}

Quagga and zebra mussels have invaded temperate lakes of eastern North America over the last 20 years. Both are benthic suspension feeders with diocy, broadcast spawning, and long-lived dispersing larvae (Mackie and Schloesser 1996; Mills et al. 1996). Although similar in life history and 
ecology, they differ markedly in their patterns of spread. After being introduced to the Great Lakes in the late 1980s (Hebert et al. 1989; Griffiths et al. 1991; May and Marsden 1992; Mills et al. 1993), zebra mussels quickly spread through temperate eastern North America whereas quagga mussels displaced zebra mussels from a few large lakes (Erie, Ontario, Michigan, and Simcoe) and there they remain (H. J. MacIsaac, Biological Sciences, University of Windsor, personal communication; Mills et al. 1999; Stoeckmann 2003; Wilson et al. 2006). Widespread transport of Dreissena can occur by boater traffic (Johnson and Carlton 1996) and has probably occurred for several years (Wilson et al. 1999). Quagga mussels may be superior competitors based on energetics (Stoeckmann 2003), but there is no satisfying explanation for their absence from surrounding smaller lakes. One clue is the apparent difference in depth adaptation: reproduction and body growth at cold temperatures favor quagga mussels (Roe and MacIsaac 1997; Claxton and Mackie 1998; Thorp et al. 1998); survival at high temperatures favor zebra mussels (Spidle et al. 1995; Thorp et al. 1998); and quagga mussels first colonized deepwater habitats before moving to shore (Mills et al. 1993; H.J. MacIsaac, personal communication). We show that source-sink dynamics between deepwater and shallow habitats, which favor quagga and zebra mussels, respectively, resolve the differences in historical spread and contemporary distributions.

\section{A Dreissenid model}

Quagga and zebra mussels, like many other aquatic invertebrates, algae, and terrestrial plants, have a sessile adult $(A)$ stage that reproduces annually and a juvenile $(Y)$ stage that disperses before settling. The lifecycle, for any species $j$ with this lifecycle can be expressed by the graph

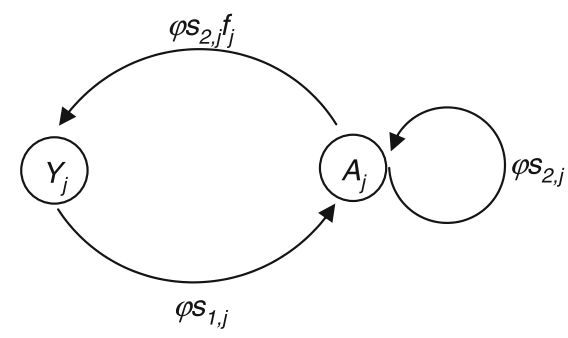

where $s_{1, j}$ and $s_{2, j}$ are the basal survival rates for juveniles and adults, respectively, $f_{j}$ is the number of juveniles produced per adult, and $\varphi$ is a function accounting for density-dependent mortality of juveniles and adults. For mussels, we assume that individuals are distributed along an environmental gradient, $x$, that, in this case, corresponds to a lake cross-section stretching along benthic habitats from the shoreline at $x=0$ to the center of the lake at $x=L$.
Dispersal links locations $x$ and $z$ according to a dispersal kernel $k(x, z)$, which results in a stage-structured integrodifference equation model

$$
\begin{aligned}
& A_{j}(x, t+1)=\varphi(x, t)\left[s_{1, j}(x) Y_{j}(x, t)+s_{2, j}(x) A_{j}(x, t)\right] \\
& Y_{j}(x, t+1)=\int_{0}^{L} \varphi(z, t) s_{2, j}(z) f_{j}(z) A_{j}(z, t) k(z, x) \mathrm{d} z
\end{aligned}
$$

where $k(x, z)$ defines the probability of moving from location $z$ to location $x$. Note that survival and fecundity are dependent on the local environment and that integration occurs over the entire domain of $x$.

Equation (17) indicates that competition is dependent on time and space, and this is based on the effects of environment and local population densities. We assume individuals compete for a limiting resource that is local relative to the scale of dispersal and so do not include a competition kernel that weights the strength of competition among individuals according to their separation distances. This is relevant, for example, to competition for nutrients or light in plants, algae, or aquatic filter feeders. We assume that competitive ability is proportional to some trait such as body size and that competition does not induce overcompensation and so choose a modified Beverton-Holt densitydependent survival term

$$
\varphi(x, t)=\frac{1}{1+\beta \sum_{j}\left[\ell_{j, y}(x) Y_{j}(x, t)+\ell_{j, a}(x) A_{j}(x, t)\right]}
$$

(Caswell 2001; Kot 2001). Here, $\beta$ is the competition coefficient that relates competitive ability to a phenotypic trait, $\boldsymbol{\ell}_{j, y}(x)$ and $\boldsymbol{\ell}_{j, a}(x)$, which we take to be shell lengths of juveniles and adults, respectively. We assume that $\beta$ is the same for each species and life-history stage and that variation in competitive ability among species and stages is accounted for in $\boldsymbol{\ell}_{j, y}(x)$ and $\boldsymbol{\ell}_{j, a}(x)$.

We compiled statistical models of mussel growth, survival, and reproduction from the literature and linked these to position along benthic habitat via a mean summer temperature profile, $T=30 \mathrm{e}^{-0.05 x}$. Details of the parameterization are given in Appendix 2 and the spatial dependency of model parameters are shown in Fig. 1. Results are qualitatively the same for most temperature functions we considered, including those with thermal stratification. Dispersal is determined by the diffusion-decay equation

$$
\frac{\mathrm{du}}{\mathrm{dt}}=D \frac{\mathrm{d}^{2} \mathrm{u}}{\mathrm{dx}^{2}}-\alpha u
$$

where $u$ is the density of larvae, $D$ is the diffusion coefficient governing dispersion of larvae and $\alpha$ is the mortality rate of larvae. We assume that at the shoreline larvae are reflected back into the lake and at $x=L$, larvae leaving the domain are replaced by larvae entering the 

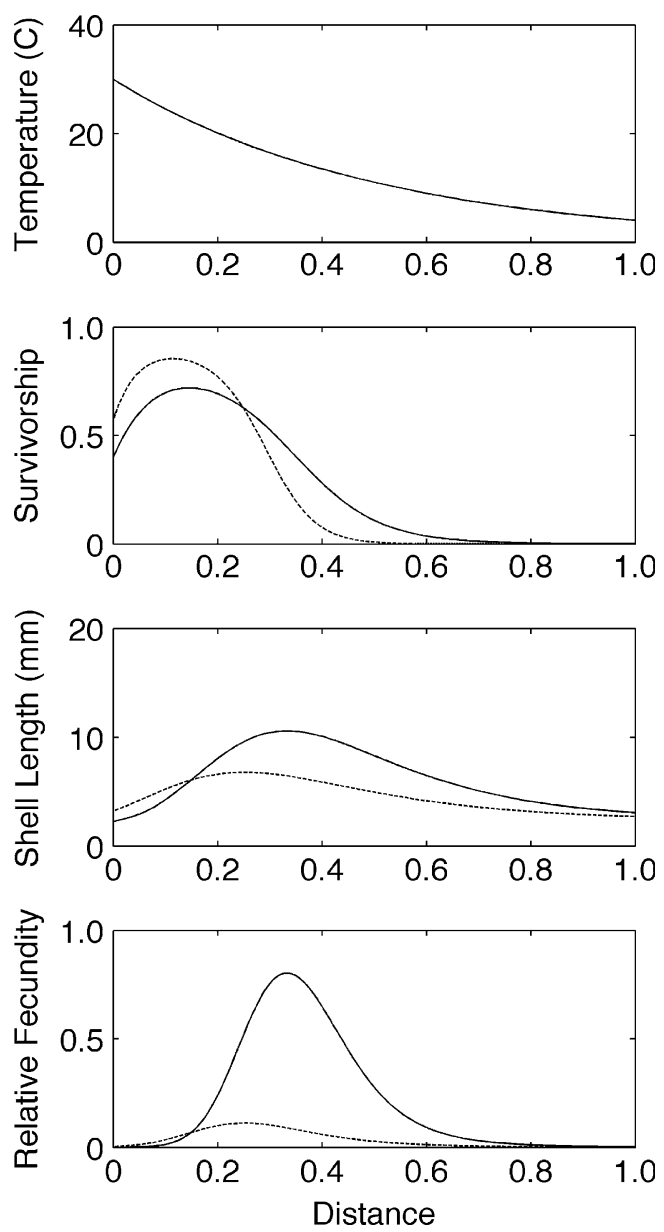

Fig. 1 Spatial dependency of temperature and quagga (solid lines) and zebra (dashed lines) mussel survivorship, shell length, and relative fecundity. Appendix 2 details the statistical submodels underlying these spatially dependent model parameters

domain from the opposite side of the lake. This leads to reflecting boundary conditions and dispersal kernel with a Fourier series representation (van Kirk and Lewis 1999).

Single species dynamics

We begin by deriving expressions for $R_{0}$ and its reproduction and survival components for the single species case by ignoring competition (by setting $\varphi=1$ ). We follow by examining the consequences of dispersal for source-sink dynamics using Eq. (4) and species persistence using Eqs. (6) and (8). The single species model without dispersal or competition is a linear matrix population model

$\mathbf{N}_{j}(x, t+1)=\mathbf{B}_{j}(x) \mathbf{N}_{j}(t)$

where

$\mathbf{B}_{j}(x)=\left(\begin{array}{cc}0 & s_{2, j}(x) f_{j}(x) \\ s_{1, j}(x) & s_{2, j}(x)\end{array}\right)$ and $\mathbf{N}_{j}$ is a vector containing the abundances of adults and juveniles. To calculate the net reproductive value, we divide $\mathbf{B}_{j}$ into transition and fecundity components, $\mathbf{B}_{j}=\mathbf{T}+\mathbf{F}$, where

$\mathbf{T}_{j}(x)=\left(\begin{array}{cc}0 & 0 \\ s_{1, j}(x) & s_{2, j}(x)\end{array}\right)$

and

$\mathbf{F}_{j}(x)=\left(\begin{array}{cc}0 & s_{2, j}(x) f_{i}(x) \\ 0 & 0\end{array}\right)$

Any founding population will produce $\mathbf{F}$ offspring in the following year. $\mathbf{T}$ of the founding population will survive to the next year and produce $\mathbf{F}$ offspring. After 2 years, $\mathbf{T}^{2}$ of the founding population will produce $\mathbf{F}$ offspring, and so on. The lifetime reproductive output of any founding individual will be $\left(\mathbf{F}+\mathbf{T F}+\mathbf{T}^{2} \mathbf{F}+\mathbf{T}^{3} \mathbf{F}+\ldots\right)=\left[\Sigma_{k} \mathbf{T}^{k}\right] \mathbf{F}=\mathbf{F}(\mathbf{I}-$ $\mathbf{T})^{-1}$ (Appendix 3). This expression is another example of a next generation operator and the net reproductive value, $R_{0}$, is its dominant eigenvalue (Cushing and Zhou 1994; Caswell 2001). For our model, the net reproductive value of a small introduced population when dispersal is ignored is

$\widehat{R}_{0}=\rho\left[\mathbf{F}(\mathbf{I}-\mathbf{T})^{-1}\right]=s_{1, j} s_{2, j}\left[f_{j}\left(1-s_{2, j}\right)^{-1}\right]=\Psi \Lambda$

which is grouped into two terms that have clear biological interpretation. The term on the right, $\Lambda=f_{j}\left(1-s_{2, j}\right)^{-1}$, is the total number of juveniles produced in the lifespan of an adult. This arises through the multiplication of an adult's annual fecundity, $f_{j}$, with its expected lifespan, $\left[1-s_{2, j}\right]^{-1}$ (which is the sum of the series $1+s_{2, j}+s_{2, j}{ }^{2}+s_{2, j}{ }^{3}+\ldots$ ). The other term, $\Psi=s_{1, j} s_{2, j}$, is the probability a juvenile is recruited into the adult population. A juvenile survives its first year with probability $s_{1, j}$ and then is recruited into the reproductive adult population with probability $s_{2, j}$. The symbol $\rho$ denotes the calculation of the dominant eigenvalue of the bracketed expression.

With the reproduction $(\Lambda)$ and survival $(\Psi)$ components of $R_{0}$ defined above for Dreissena, we used Eqs. (3), (4), and (8) to map fundamental niche space, source and sink regions, and calculate global persistence criteria. Figure 2 shows how dispersal interacts with reproduction and survival terms to affect source-sink dynamics and global persistence criteria. When zebra and quagga mussels exist in isolation, dispersal can affect the spatial distribution of source and sink regions as well as global persistence criteria. There are sink regions near shorelines, source regions in shallow to intermediate depth, and sink regions in deep habitats for both species. As dispersal increases, nearshore habitats switch from sink to source function because the shoreline is a reflecting boundary near source habitat so a large proportion of progeny produced near the 
Fig. 2 Effects of dispersal on source-sink dynamics, spatial distributions, and persistence criteria of single species dynamics of quagga and zebra mussels. Top panels show spatial distributions of $R_{0}$ components - reproductive output $\Lambda(x)$ and survival $\Psi(x)$. These components are then connected by dispersal according to the dispersal kernel, $k(x, z)$, whose probability density is plotted at $z=0.5$ for three different kernels (thick gray lines with parameters $L=10,0.4$, 0.05 where $L$ is the nondimensionalized parameter in equation 10 in van Kirk and Lewis 1999, which measures the characteristic dispersal distance relative to lake size, rather than the habitat length of our model). The resulting stable spatial distribution of population density, $\phi(x)$ (dashed lines), is plotted for each corresponding dispersal kernel. Source-sink dynamics are described by the thin lines in the bottom three rows of panels, where the division between source and sink, $R_{0}(x)=1$, is shown by the horizontal dotted lines. Sources are regions where $R_{0}(x)>1$ and sinks are regions where $R_{0}(x)<1$. The source-sink dynamics also scale up to affect global persistence criteria, $\bar{R}_{0}$, which are shown in each panel. The shore is at $x=0$ and the lake's center is at $x=1$
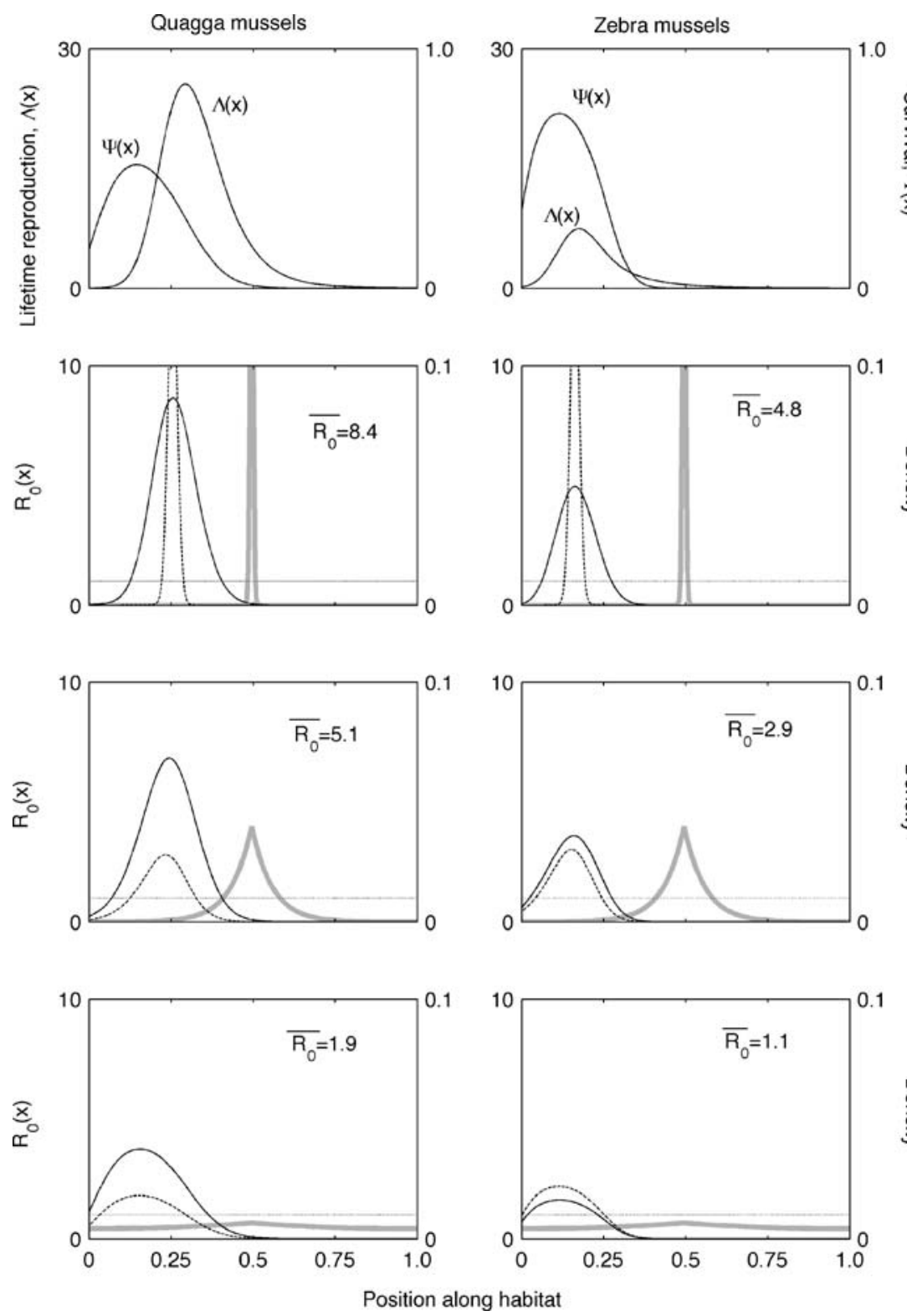

shoreline get recruited into favorable habitat at shallow to intermediate depth. The opposite effect occurs near the deepwater boundary between source and sink regions. There, as dispersal increases and connects source and sink habitats the deepwater edge of source habitats decline in their function as sources and the sink regions moves slightly into shallower depths. At a lake-wide scale, the global net reproductive value declines as dispersal increases (but remains greater than unity) because of increased losses to extensive sink regions in deepwater habitats. Examination of the stable population distribution, $\phi(x)$, and the equilibrium distributions reveal that, at low dispersal, the invading population remains concentrated in the most favorable habitats but then spreads out as dispersal increases (Fig. 2).
Competing species dynamics

Consider two competing species described by Eq. (17) where the species differ in their environmental response. Of interest here are the interactions among competitive interactions, differential environmental response, and dispersal in determining source-sink dynamics and species coexistence. We use a mutual invasibility analysis to evaluate the conditions under which coexistence and/or exclusion results from competition. The logic is that competing species can coexist if each species, when rare, can invade the resident community. The analysis entails first calculating the equilibrium densities of a resident species with no interspecific competition and, second, calculating the net reproductive value of the invader as it 
experiences competition with residents. The invader is considered to be sufficiently rare to escape intra-specific competition but it may be excluded by interactions with resident competitors. Details of the competition dynamics, given by Eq. (18), make the system monotone and sublinear. Hence, one-way invasibility (one species can invade the second, but the second cannot invade the first) will result in a globally stable equilibrium with only one species present, and hence competitive exclusion.

We begin by simplifying the model by rescaling the state variables. Then we investigate the effects of competition and dispersal on source-sink dynamics, species distributions, and global coexistence. First, we simplify the model by non-dimensionalizing the state variables such that $y_{i}=\beta \ell_{i, y} Y_{j}, a_{j}=\beta \ell_{j, a} A_{j}$ where $\ell_{i, y}$ and $\ell_{i, a}$ are the phenotypic traits (e.g., body size) determining competitive ability of species $j$ juveniles and adults, respectively. The survivorship and fecundity terms are already dimensionless and they remain unchanged and so $\mathbf{B}_{j}$ also remains unchanged. The competition term is simplified to

$\varphi(x, t)=\frac{1}{1+\sum_{j}\left[y_{j}(x, t)+a_{j}(x, t)\right]}$

where $j$ is summed over species. To complete the rescaling, we define $\mathbf{n}_{j}$ as the vector containing $y_{j}$ and $a_{j}$.

The invasibility analysis begins with finding the equilibrium densities of a resident species when it exists in isolation, $\mathbf{n}_{r}^{*}=y_{r}^{*}, a_{r}^{*}$, where the subscript $r$ signifies their resident status in the lake. The invader, $i$, competes with the resident, $r$, for a limiting resource, and this interaction may be sufficiently strong to prevent establishment. We are interested in the net reproductive value of the invader, but it must account for competitive interactions with residents. Because the resident is at equilibrium and the invader is at low population density, competition will reduce survivorship of the invader by

$\varphi_{i}=\frac{1}{1+y_{r}^{*}+a_{r}^{*}}$

and the population dynamics of the invader will be described by Eq. (17) with $\varphi=\varphi_{i}$. From here, we can quickly see the effect of competition on realized niche space. Recall that the fundamental niche of a species is defined by areas where $\widehat{R}_{0}(x)>1$. Because the terms in $\mathbf{B}$ are now weighted by $\varphi_{i} \leq 1$ the realized niche will be less than or equal to the fundamental niche (Figs. 3, 4). This can be seen by calculating the net reproductive value of the invader in the absence of dispersal

$\widehat{R}_{0}^{i}=\underbrace{\left(\frac{f_{i}}{1-s_{2, i}\left(1+y_{r}^{*}+a_{r}^{*}\right)^{-1}}\right)}_{\text {lifetime reproductive output }} \underbrace{\left(\frac{s_{1, i} s_{2, i}}{\left(1+y_{r}^{*}+a_{r}^{*}\right)^{2}}\right)}_{\text {survival }}$.

As resident equilibrium densities of the invader increase from zero, $\widehat{R}_{0}^{i}$ declines. The convenient separation of the net reproductive value into spatially independent reproduc-
Fig. 3 Effects of competition on quagga (solid lines) and zebra (dashed lines) equilibria and local net reproductive values in the absence of dispersal. Equilibria and net reproductive values are plotted for species in isolation $(\mathbf{a}-\mathbf{b})$ and for species in competition $(\mathbf{c}-\mathbf{d})$. The horizontal dotted line at $R_{0}(x)=1$ distinguishes locations that can support a population $\left(\widehat{R}_{0}(x)>1\right)$ from those that cannot $\left(\widehat{R}_{0}(x)>1\right)$ when the effects of dispersal are ignored. The shore is at $x=0$ and the lake's center is at $x=1$
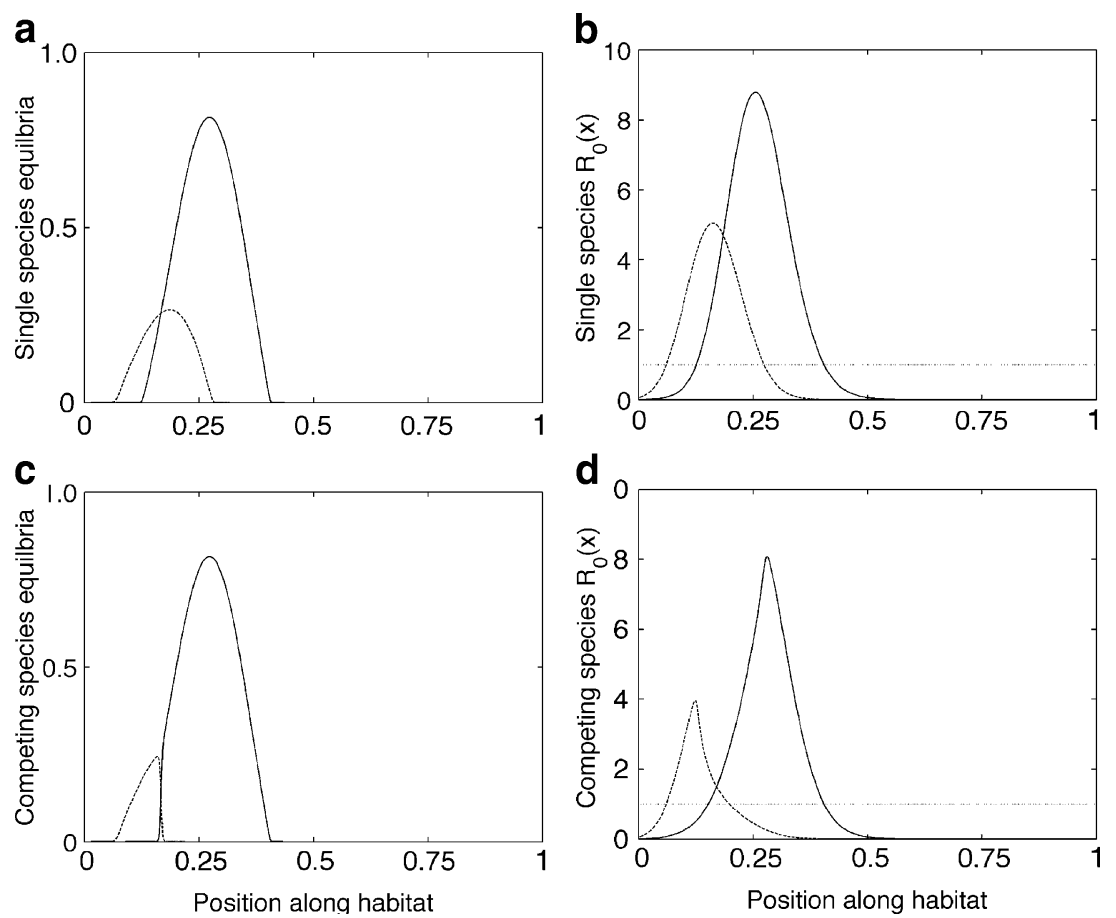

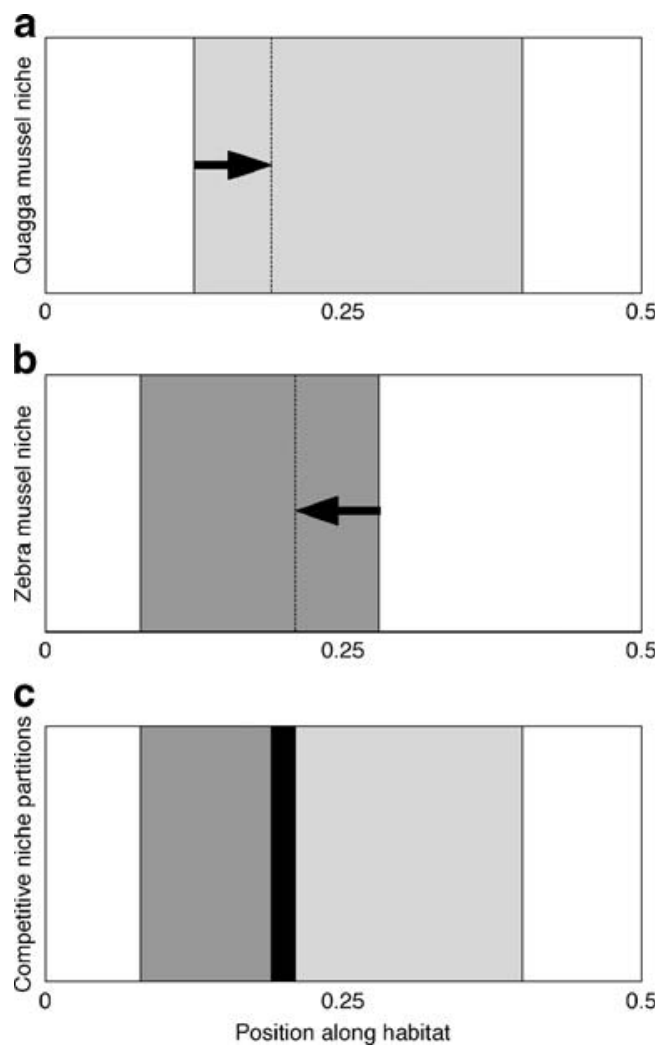

Fig. 4 Effects of competition on niche space of quagga (a, light gray) and zebra (b, dark gray) mussels in the absence of dispersal. Shaded areas in (a)-(b) represent fundamental niche space (defined by areas where $\widehat{R}_{0}(x)>1$ ) and the arrows indicate a competition-induced shift in niche boundaries to the dashed lines. Competition results in zonation of species distributions (c) with quagga mussels (light gray) occupying shallow habitats, zebra mussels (dark gray) occupying deep habitats, and a small area of coexistence (black) at intermediate depth. The shore is at $x=0$ and the lake's center is at $x=1$

tion and survival components remains. By defining the net reproductive value through the spatially separated processes of reproduction

$\Lambda^{i}(z)=\frac{f_{i}(z)}{1-s_{2, i}(z)\left[1+y_{r}^{*}(z)+a_{r}^{*}(z)\right]^{-1}}$

and survival

$\Psi^{i}(x)=\frac{s_{1, i}(x) s_{2, i}(x)}{\left[1+y_{r}^{*}(x)+a_{r}^{*}(x)\right]^{2}}$

we can make the same calculations as in the single species case, including source and sink regions $\left(R_{0}^{i}(x)\right.$ for Eq. (4)), and global persistence criteria $\left(\bar{R}_{0}^{i}\right.$ for Eq. (8)). The mutual invasibility analysis determines species coexistence when $\bar{R}_{0}^{i}>1$ for both species, exclusion occurs when $\bar{R}_{0}^{i}>1$ for one species but $\bar{R}_{0}^{i}<1$ for the other, and when $\bar{R}_{0}^{i}<1$ for both species then dominance depends on initial conditionsthe first species to reach equilibrium excludes the other.
Here, we see how species can be extirpated from locally favorable habitat $\widehat{R}_{0}(x)>1$, even if it remains a source location $R_{0}^{i}(x)>1$, if the global net reproductive value is less than one $\bar{R}_{0}^{i}(x)<1$. One can directly map the sourcesink dynamics that underlie all these scenarios of exclusion and coexistence (Figs. 5, 6).

It is evident from Fig. 2 that zebra mussels and quagga mussels overlap substantially in fundamental niche space. This is detailed by overlapping distributions of single species equilibria and regions of suitable habitat (where $\left.\widehat{R}_{0}(x)>1\right)$. However, there were also interspecific differences. The peak single species equilibria of quagga mussels occurred at a greater depth and distance from shore than it did for zebra mussels. Further, the equilibrium abundance of quagga mussels was greater than zebra mussels for the deeper part of the lake but in the shallows zebra mussels attained higher densities than quagga mussels. Competition induced a shift in equilibria and regions of suitable habitat such that zebra mussels occupied nearshore depths and quagga mussels occupied deepwater habitats. This competition-induced shift from fundamental to realized niche is summarized in Fig. 4 which shows the deepwater boundary for zebra mussels shifting to shallower habitats and the shallow water boundary for quagga mussels shifting to greater depths. This effect corresponds nicely with the expected zonation of species distributions given the relative adaptations of quagga mussels to deepwater habitats and zebra mussels to shallow habitats. Finally, there remained a narrow band of coexistence where the ranges of the competing species met.

There were complex interactions among dispersal, competition, and habitat size that affected source-sink dynamics which departed strongly from the mapping of each species' fundamental niche (Fig. 4). For example, the entire fundamental niche of a species can become a sink. This is exemplified by zebra mussels in large lakes (Fig. 5), where dispersal coupling to large regions of unsuitable deepwater habitat was sufficiently strong to turn the zebra mussel fundamental niche into a sink. The effect was also evident for quagga mussels in small lakes where the fundamental niche declined sharply in its function as a source (Fig. 6). These effects can be understood first through competition inhibiting reproduction and survival, second by dispersal coupling suitable and unsuitable habitat, and finally by habitat size regulating the production value of source habitat and the absorptive value of sink habitat (Figs. 5, 6). Dispersal also acted to produce source regions outside a species' fundamental niche, in particular nearshore habitats, for the same reasons as described in the single species case; there, competition was not sufficiently strong to counteract the effects of dispersal because single species equilibria of residents were low. When dispersal was relatively local, there was global competitive coexis- 
Fig. 5 Effects of competition and dispersal on source-sink dynamics and $R_{0}^{i}(x)$ components in a large lake. As dispersal increases among columns from left to right, the responses in the spatial distributions are plotted for single species equilibria, survival $\Psi(x)$, lifetime reproductive output $\Lambda(x)$, spatial net reproductive values for the invader $R_{0}^{i}(x)$, and equilibria of competing species. Solid lines are quagga mussels and dashed lines are zebra mussels. Regions where $R_{0}^{i}(x)>1$ function as sources and $R_{0}^{i}(x)<1$ function as sinks for the invader. The horizontal dotted line in the bottom row of panels corresponds to $R_{0}(x)=1$. Shaded areas correspond to fundamental niche space (panel rows 5-6) and zones of dominance at equilibria (bottom row of panels) for quagga (light gray) and zebra (dark gray) mussels. The shore is at $x=0$ and the lake's center is at $x=1$
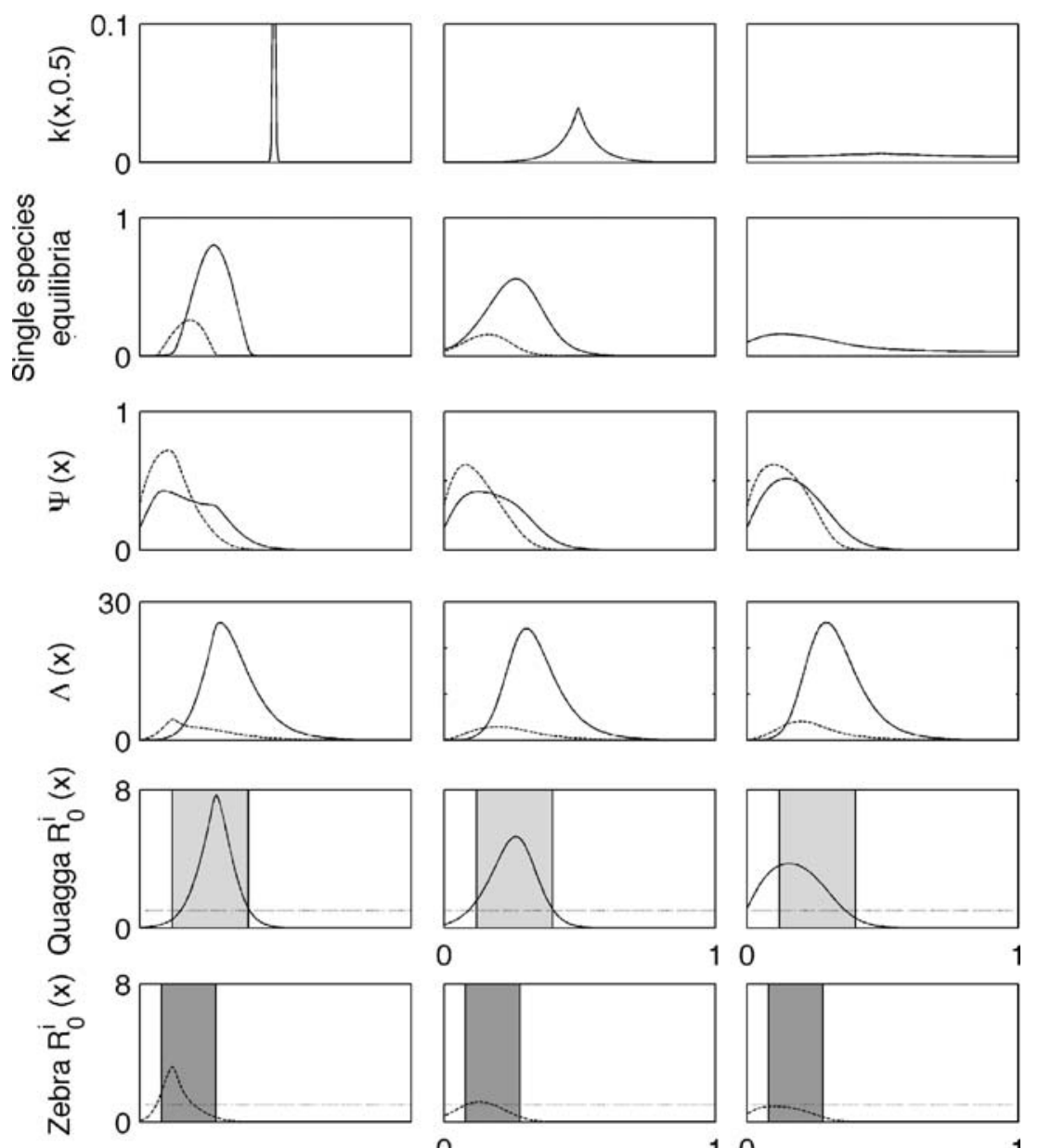

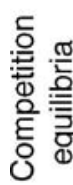

0

10

1
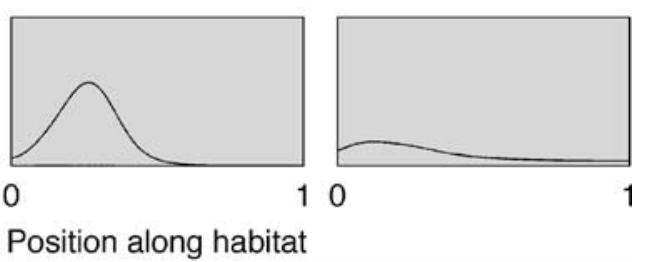

tence through niche partitioning such that zebra mussels dominated shallow habitats and quagga mussels dominated deepwater habitats. As dispersal increased, species zonation was lost and the species with the greatest amount of favorable habitat dominated the entire spatial domain (Figs. 5, 6).

The combined effects of dispersal, competition, and habitat size on global exclusion and coexistence are summarized in Fig. 7. When dispersal was local, there was a large region of global coexistence which was characterized by zonation-zebra mussels occupied shallow habitats and quagga mussels occupied deep habitats. However, as dispersal coupling between shallow and deepwater habitats increased, coexistence declined, and the dynamics moved towards lake-wide domination by one species. The superior species was determined by the relative amount of favorable habitat: quagga mussels occupied large lakes with extensive deep/cold habitat and zebra mussels occupied small lakes where there was a larger proportion of shallow/warm habitat (Fig. 7). This effect can be understood by the underlying source-sink dynamics (Figs. 5, 6), where under extensive dispersal, the relative amounts of favorable habitat determine the source or sink functioning of fundamental niche space of each species. These effects scale up to a metapopulation distribution where quagga mussels occupy large lakes and zebra mussels occupy small lakes. This distribution is consistent with the observed spread and present distributions of quagga and zebra mussels across temperate eastern North America. 
Fig. 6 Effects of competition and dispersal on source-sink dynamics and $R_{0}^{i}(x)$ components in a small lake $(20 \%$ of the lake in Fig. 5). The shore is at $x=0$ and the lake's center is at $x=0.2$. See Fig. 5 caption for details
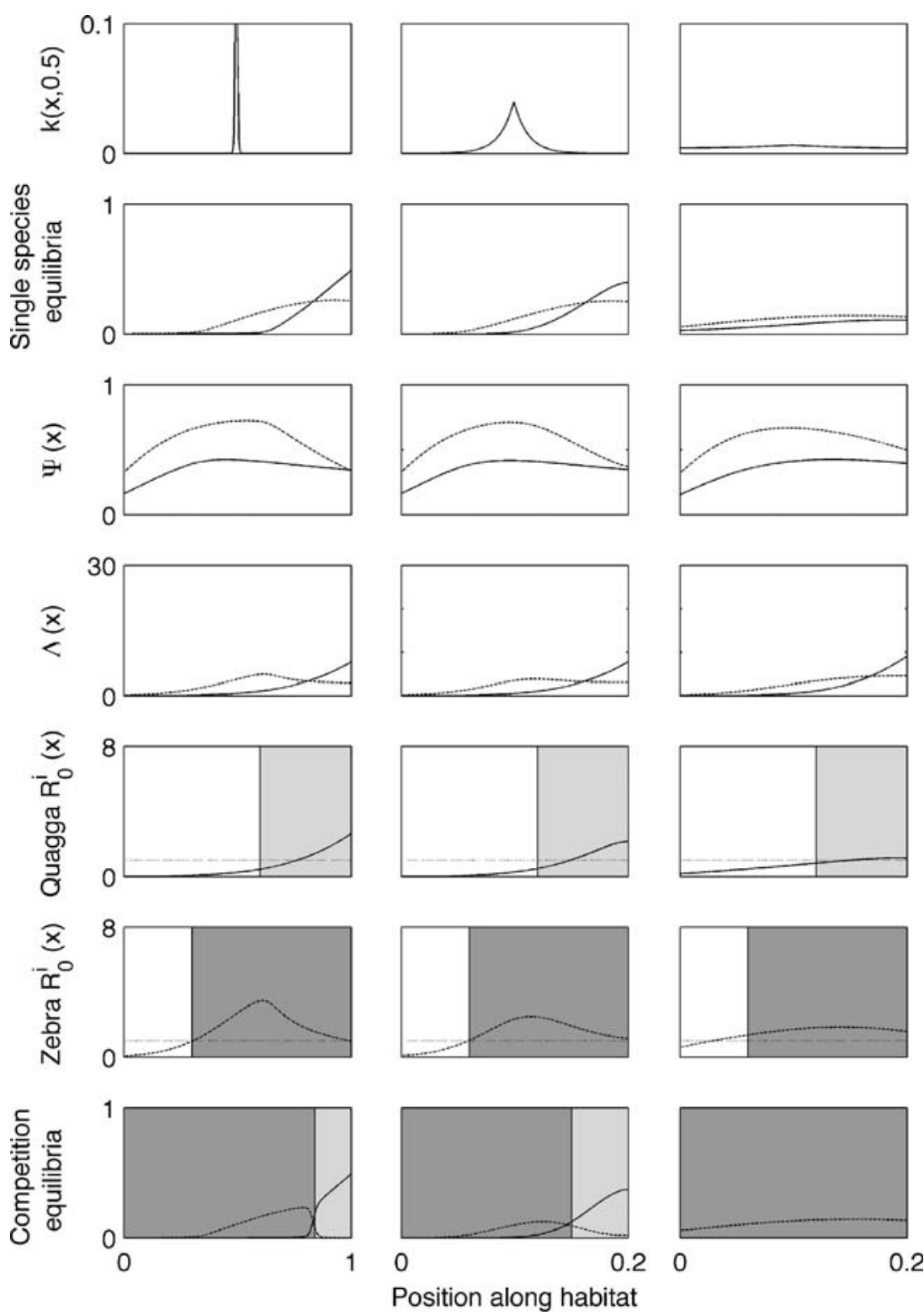

\section{Discussion}

The $R_{0}$ theory for source-sink dynamics can accommodate a diversity of spatial population models and provides a quantitative means for relating niche, source-sink dynamics, and species distributions. Insights come from an ability to track the influence of environmental heterogeneity, biotic interactions, and dispersal on source-sink dynamics. Traditional approaches to source-sink dynamics classified habitat patches according to the local population growth rate in the absence of dispersal (Pulliam 1988) whereas recent advances classify sources and sinks according to the contribution a local patch makes to the metapopulation (Figueira and Crowder 2006; Runge et al. 2006). The contribution metric, $C^{r}$, provides a general means for classifying patches as sources and sinks (Runge et al. 2006), but limitations include a loss of biological interpretation from the calculations, rapidly accelerating complexity of the calculations, and no application for continuous landscapes or competitive coexistence. For resource competition models, $R^{*}$ theory provides a means for predicting the outcome on species persistence (Tilman 1982), but the theory breaks down when dispersal is introduced (Abrams and Wilson 2004). In spatial competition models, there are often model-specific criteria for coexistence and exclusion, which are sometimes difficult to interpret biologically (Snyder and Chesson 2004). Among spatial competition models, there has been no general framework for classifying sources and sinks according to ideas of contribution metrics (Runge et al. 2006) or for quantifying the relations among niche, dispersal, competition, and distribution. The $R_{0}$ source-sink theory overcomes many of these limitations. It is broadly applicable to single and competing 

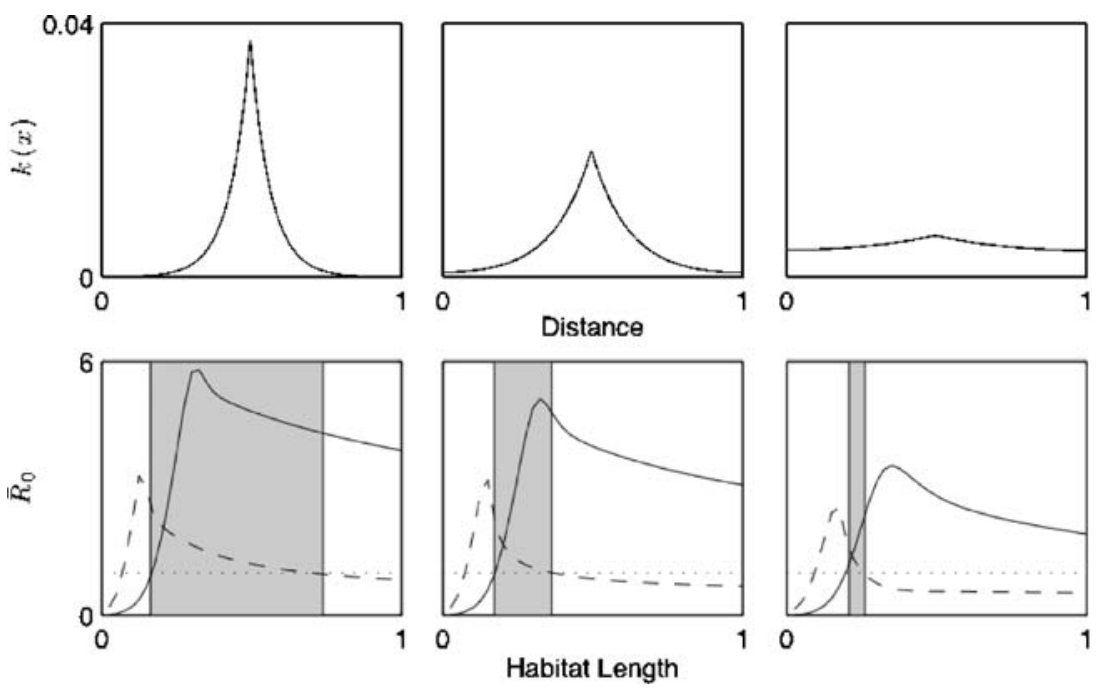

Fig. 7 Declining coexistence (shaded regions) of quagga and zebra mussels with increasing dispersal. Dispersal increases among panel columns from left to right and is represented by the dispersal kernel $k(x, z)$ plotted for $z=0.5$. In the second row of panels, species persistence (determined by the global net reproductive value $\bar{R}_{0}^{i}$ ) is plotted against habitat size (not position along habitat) for quagga (solid lines) and zebra (dashed lines) mussels for the corresponding

species models. The calculations are biologically intuitive. It is applicable to patch metapopulations or metacommunities as well as on continuous landscapes. Finally, it provides a general framework for source-sink dynamics in the relation between niche and distribution.

However, the $R_{0}$ theory for source-sink dynamics becomes more complex when individuals can both reproduce and disperse for many years, for example, giving a population dynamics model of the form

$$
\begin{aligned}
n(x, t+1)= & \int s(n(z, t), z) n(z, t) k_{\mathrm{A}}(z, x) \mathrm{dz} \\
& +\int f(n(z, t), z) s(n(z, t), \mathrm{z}) n(z, t) k_{J}(z, x) d \mathrm{z}
\end{aligned}
$$

(compare with Eq. (13)). In this situation, the formulae for $R_{0}$ become complicated by the need to integrate annual dispersal and spatially dependent survival and reproduction over the lifetime of an individual (Appendix 4). This is relatively easy if the lifecycle has a discrete number of years, but if there is simply an annual survival probability followed by dispersal, such as in Eq. (30), the calculations become complex. We leave this situation for future mathematical development.

To see the utility and limitations of $R_{0}$ theory, it is useful to apply it to previous theory in source-sink dynamics. The Pulliam (1988) source-sink model, in its linearized form, is

$n(x, t+1)=\sum_{z}\left(P_{\mathrm{A}}(z)+P_{\mathrm{J}}(z) \beta(z)\right) n(z, t) k^{\prime}(z, x)$ dispersal kernel. The invader can establish in lakes where $\bar{R}_{0}^{i}>1$ and coexistence occurs in those lakes where $\bar{R}_{0}^{i}>1$ for both species. The horizontal dotted line in the bottom row of panels marks $\bar{R}_{0}^{i}=1$. Note that the region of lake sizes permitting coexistence (shaded regions) declines with increasing dispersal leading to small lakes occupied by zebra mussels and large lakes occupied by quagga mussels. The shore is at $x=0$ and the lake's center is at $x=1$

where $x$ denotes patch identity, $P_{\mathrm{A}}(z)$ is adult survival in patch $z, P_{\mathrm{J}}(z)$ is juvenile survival in patch $z, B(z)$ is the number of juveniles produced per adult in patch $z$, and $k^{\prime}(z, x)$ is the probability an individual disperses from patch $z$ to patch $x$ in 1 year. Pulliam (1988) used the local reproductive rate $\lambda(x)=P_{\mathrm{A}}(x)+P_{\mathrm{J}}(x) \beta(x)$, Eq. (1), to identify source patches $\lambda(x)>1$ and sink patches $\lambda(x)<1$. The analogous quantity to the local reproductive rate in $R_{0}$ theory is the local net reproductive value, or niche $R_{0}(x)$, which has the lifetime reproductive output component $\Lambda(x)=\beta(x)\left[1-P_{\mathrm{A}}(x)\right]^{-1}$ and survival term $\Psi(x)=P_{\mathrm{J}}(x)$ giving $\widehat{R}_{0}(x)=\Lambda(x) \Psi(x)=\beta(x) P_{\mathrm{J}}(x)\left[1-P_{\mathrm{A}}(x)\right]^{-1}$. It is easy to verify that $\lambda(x)=1$ implies $\widehat{R}_{0}(x)=1, \lambda(x)<1$ implies $\widehat{R}_{0}(x)<1$, and $\lambda(x)>1$ implies $\widehat{R}_{0}(x)>1$. However, note that Pulliam's model (Eq. (31)) similar to Eq. (30) in that there is annual survival, dispersal, and reproduction of adults. Hence, it suffers the same difficulty in calculating $\widehat{R}_{0}(x)$ and $\bar{R}_{0}$ easily, because one must track the spatial lifecycle trajectory of each individual starting at some location $z$ when calculating lifetime reproductive output (Appendix 4).

The $R_{0}$ theory developed for the Dreissena competition model assumes an absence of depensation or Allee effects, which, if included, may change predictions on exclusion and coexistence based on a mutual invasibility analysis (Boldin 2006). Extension of our framework to include Allee effects is an area for future work. We also did not consider matrix population models where different population stages have different dispersal kernels. This is another 
mathematical challenge for future developments in sourcesink theory. The model we considered in detail had sessile adults and a single dispersal event associated with production of offspring. For this kind of model, $R_{0}$ theory provides biologically pleasing calculations. The $R_{0}$ theory for source-sink dynamics may not be the simplest framework for studying spatial population dynamics for all types of models. However, our results indicate that the theory is broadly applicable and may provide a general framework for studying source-sink dynamics under many model types on fragmented and continuous landscapes.

In the analysis of competition between zebra and quagga mussels, the $R_{0}$ source-sink theory showed how environment, dispersal, and habitat size can influence the distributions of source and sink habitat and the resulting species distributions. In this case, an environmental gradient created variation in habitat quality for the competing species. Dispersal across habitats of varying size and quality then affects the local and global outcome of competition. Environmental gradients have been shown, in other studies using cellular automata, to affect the distributions of single species as well as competitive and cooperative communities (Wilson et al. 1996; Wilson and Nisbet 1997). However, only recently have environmental gradients been considered to produce source-sink dynamics that affect species distributions in nature (Rex et al. 2005). While (Rex et al. 2005) hypothesized that source-sink dynamics along depth gradients may regulate biodiversity in the deep sea benthos, it is immediately apparent that environmental gradients are ubiquitous, from the deep sea to the marine intertidal to elevation gradients in mountain ranges to latitudinal gradients across continental landscapes. The $R_{0}$ source-sink theory provides a framework for quantitatively characterizing source-sink dynamics and spatial competition on such continuous landscapes.

Consistent with other studies, our analysis showed that competition can reduce a species range (Case et al. 2005), and that dispersal can cause species distributions to differ from fundamental niche space due to immigration from source populations (the rescue effect) (Brown and KodricBrown 1977; Pulliam 1988) or emigration to sink populations (Amarasekare and Nisbet 2001). Underlying these effects were changes in source and sink regions that deviated from fundamental niche space due to dispersal between and reflecting boundaries near favorable or unfavorable habitat. Competition enhanced these effects on source and sink regions, to the extent that an entire fundamental niche can become a sink through strong dispersal coupling to unsuitable habitat. At a global (whole-lake) scale, coexistence was enhanced by local dispersal that maintained source and sink regions, and resulted in niche partitioning through species zonation.
Similar to other source-sink models (Amarasekare and Nisbet 2001, Snyder and Chesson 2004), large-scale dispersal had a homogenizing effect. This either decreased or increased source regions depending on the relative amounts of favorable and unfavorable habitat and resulted in a single species - the one with the greatest amount of favorable habitat - dominating the entire domain.

The source-sink dynamics underlying zebra and quagga mussel competition resolve a long-standing paradox in invasion biology. The invasion of North America by Dreissena began with an initial rapid spread of zebra mussels across most of the temperate East followed by the appearance of quagga mussels and their subsequent displacement of zebra mussels in a few large lakes but not in surrounding smaller lakes (Mills et al. 1996, 1999; Wilson et al. 2006; HJM, personal communication). The estimated fundamental niches of quagga and zebra mussels overlapped substantially indicating that competitive exclusion should apply, as others like Stoeckmann (2003) have suggested. Quagga mussels may have an energetic advantage (Stoeckmann 2003) but this fails to explain their absence from surrounding smaller lakes. Dispersal limitation is not an adequate explanation either because Dreissena are readily transported by the same vectors (boat traffic) or this has probably occurred for many years (Johnson and Carlton 1996; Wilson et al. 1999). Closer inspection of quagga and zebra mussel fundamental niches reveals subtle interspecific differences - quagga mussels are better adapted to deeper habitats and zebra mussels are better adapted to shallower habitat. This subtle difference in adaptation, measured directly as fitness through $R_{0}$, leads to sourcesink dynamics between deepwater and shallow habitats that resolves the paradox of Dreissena competition. Quagga mussels are predicted to exclude zebra mussels from large deep lakes and zebra mussels are predicted to exclude quagga mussels from small shallow lakes.

Differences in depth adaptation between quagga and zebra mussels are explained by subtle interspecific differences in survival and growth responses to temperature (Thorp et al. 1998) and scaling relations between body size and fecundity (Walz 1978; Stoeckel et al. 2004; Strayer and Malcom 2006). Competition alone induced niche partitioning by narrowing each species' realized niche into a band of quagga mussels in deep habitats, a narrow band of coexistence at intermediate depth, and a band of zebra mussels in shallow habitats. Competitive interactions were assumed to be mediated by body size - a passive form of interference competition where smaller mussels can only access food resources in the water column that have been partially pre-filtered by larger mussels. When we introduced dispersal at local scales, these patterns of species zonation remained over a broad range of lake sizes. 
However, Dreissena have vast dispersal potential owing to long-lived planktonic larvae (Mackie and Schloesser 1996; Mills et al. 1996). As dispersal increased the range of lake sizes capable of supporting both species declined; patterns of species zonation were lost and a single species typically dominated the entire lake. The partitioning of lake-wide dominance to quagga or zebra mussels depends on an assumption that larger lakes have a larger proportion of colder benthic habitat and that smaller lakes have a larger proportion of warmer benthic habitat during summer. Since lakes typically have a thermal depth gradient during summer, either continuous or discontinuous, this causes shallow benthic habitats to be warmer than deepwater benthic habitats. On average then, larger lakes would have a larger proportion of deepwater and colder benthic habitat that favors Quagga mussels. These effects scale to predict a metacommunity distribution where quagga mussels dominate large lakes and zebra mussels dominate small lakes, which is consistent with their patterns of spread and present distributions.

The classical result for source-sink dynamics and spatial competition is that source-sink dynamics among habitat patches support the coexistence of competing species (Levin 1974; Amarasekare and Nisbet 2001). The landscape coexistence of zebra and quagga mussels has a different mechanism-source-sink dynamics within lakes undermine within-lake coexistence. Subtle differences in fitness between zebra and quagga mussels in response to temperature creates a source-sink dynamics along the continuous habitat of a lake bottom. Competitive interactions with quagga mussels yield source habitat for zebra mussels in shallow habitats and sink habitat in deep habitats. In contrast, competition with zebra mussels leads to sink regions in shallow habitat and source regions in deep habitats for quagga mussels. Lake size then mediates the outcome of source-sink dynamics between deepwater and shallow habitats. Because dispersal is large, coupling between the two habitat types is strong, leading to dominance of the species with the greatest amount of habitat and exclusion of the species with the least. Dominance of one species and exclusion of the other depends on lake size, where strong connectivity to competition-determined sink habitat extinguishes species from what is otherwise suitable habitat, a result theorized previously by Amarasekare and Nisbet (2001) and Schreiber and Kelton (2005). The among-lake pattern of coexistence arises then because the landscape includes small lakes where zebra mussels prevail and large lakes where quagga mussels prevail, not because of source-sink dynamics among lakes.

While the relative adaptations to deep and shallow habitats for quagga and zebra mussels are well supported, there are some factors we did not consider. For example, temperature control of gametogenesis and spawning allows reproduction by quagga mussels at greater depths than zebra mussels (Roe and MacIsaac 1997; Claxton and Mackie 1998). There are also interspecific differences in adaptations to wave swept environments that may favor zebra mussels in shallow habitats (Mills et al. 1993). These factors would act to increase the interspecific partitioning of lakes into source and sink regions and further substantiate our results. Other factors may act in neutral or unknown ways. For example, mussels can deplete food supply at whole-lake scales (Bridgeman et al. 1995; Fahnenstiel et al. 1995; Idrisi et al. 2001), which implies that competition may occur among individuals other than immediate neighbors. Further, if food limitation induces interspecific differences in survival, growth, or fecundity, then this could affect the model results. These types of differences, if they exist, could be directly incorporated into the model if they are sufficiently documented over a range of temperatures. Temperature was incorporated in the model as a mean summer spatial profile and we did not consider withinseason temporal variation. This assumes that competitive interactions, growth, and reproduction occur primarily during summer months. Further, we used data from mesocosm experiments to parameterize temperature dependency of survival and growth (Thorp et al. 1998) and these of course depart from field conditions. Nevertheless, while the model made many simplifying assumptions, we believe it captured the essential features of Dreissena biology - in particular interspecific differences in habitat adaptationand allowed us to examine how these differences interact with dispersal, competition, and habitat size to produce source-sink dynamics and explain species distributions.

Source-sink dynamics may be ubiquitous in nature. They may occur along depth gradients and regulate biodiversity in the deep sea benthos (Rex et al. 2005). They may occur along depth gradients in lakes and structure benthic communities, as we have suggested here. Source-sink dynamics may occur on other environmental gradients down intertidal shorelines, up mountain ranges, or across continental scales. For fragmented habitats, sourcesink dynamics among habitat patches may facilitate coexistence of competing species (Levin 1974; Amarasekare and Nisbet 2001). The general source-sink theory we have presented here can accommodate these various spatial structures yet yield general and coherent criteria that are biologically intuitive for understanding relationships among niche, dispersal, biotic interactions, source-sink dynamics, and species distributions. It is important to note that our methods are not limited to theory but can be directly ported to Geographic Information Systems to accommodate environmental information from real habitats. The application of source-sink theory to competition between quagga and zebra mussels resolves their paradoxical distributions and shows that source-sink dynamics rather than dispersal 
limitation explain the absence of species from suitable habitat. There are numerous other possible applications of our theory, perhaps most notably spatial coexistence, habitat conservation, marine protected areas, and ecological responses to climate change.

Acknowledgements We thank Franz Weissing, William Wilson, Brad Anholt, Marjorie Wonham, Hugh MacIsaac, and David Lodge for helpful discussions and comments. We are grateful to Hugh MacIsaac and Ed Mills for updating us on the distributions of Dreissena and to James Thorp for sharing parameter values of statistical models. This work was supported by the US National Science Foundation grant DEB 02-13698, by the Natural Science and Engineering Research Council of Canada Collaborative Research Opportunities program, by the Canadian Aquatic Invasive Species Network, and by a Canada Research Chair to MAL.

\section{Appendix 1}

Here we show how Eq. 8, which applies to the general case, can be used to find intuitively pleasing solutions to two special cases: when the initial individual is introduced at one location and when dispersal is uniform across the domain.

For the point release, we must introduce the Dirac delta function, $\delta(z-x)$, which is a distribution (a) equals zero for all $x \neq z$, (b) is unbounded when $x=z$, and (c) integrates to unity. For example, if an individual disperses according to the Dirac delta function, it will remain where it is probability one-it does not disperse. Similarly, if the initial individual is introduced at some location $\xi$, then it has an initial distribution $\phi_{0}(x)=\delta(x-\xi)$. So if we consider an adult individual that is introduced at location $\xi$, Eq. (6) simplifies to

$$
\begin{aligned}
\Gamma \delta(x-\xi) & =\Psi(x) \int_{0}^{L} \Lambda(z) \delta(z-\xi) k(\xi, z) \mathrm{d} \mathrm{z} \\
& =\Psi(x) \Lambda(\xi) k(\xi, x)
\end{aligned}
$$

We define $R_{0}(x)$ to be the total number of offspring produced at any spatial location by the single individual by integrating Eq. (32) over all spatial locations $x$ to yield

$$
\begin{aligned}
R_{0}(\xi) & =\int_{0}^{L} \Psi(x) \Lambda(\xi) k(\xi, x) \mathrm{dx} \\
& =\Lambda(\xi) \int_{0}^{L} \Psi(x) k(\xi, x) \mathrm{d} \mathrm{x}
\end{aligned}
$$

as given in Eq. (4). In the case where there is no dispersal $k(\xi, x)=\delta(x-\xi)$ and Eq. (33) simplifies to Eq. (3).
When dispersal operates on the largest scale possible, the dispersal kernel becomes a uniform distribution. The lake is essentially homogenized by dispersal so that larvae can settle anywhere with equal probability regardless of where they were released. In this case, the next generation operator, Eq. (4), becomes

$\Gamma \phi(z)=\Psi(z) \frac{1}{L} \int_{0}^{L} \Lambda(x) \phi_{0}(x) \mathrm{dx}$.

If we choose $\phi(z)=\psi(z)$, then we see that $\psi(z)$ is the eigenfunction and that the dominant eigenvalue is

$$
\begin{aligned}
\bar{R}_{0} & =\frac{1}{L} \int_{0}^{L} \Lambda(x) \Psi(x) \mathrm{dx} \\
& =\frac{1}{L} \int_{0}^{L} R_{0}(x) \mathrm{dx}
\end{aligned}
$$

which is pleasingly intuitive. When dispersal operates on a large enough scale, the lake-wide net reproductive rate, $\bar{R}_{0}$, becomes the spatial average of the local $R_{0}(x)$.

\section{Appendix 2}

In this section, we describe the Dreissena model parameterization. Equation (9) is our model with two speciesquagga and zebra mussels. We compiled statistical models of mussel growth, survival, and reproduction from the literature and linked these to position along benthic habitat from the shore at $x=0$ to the center of a lake at $x=L$ via a mean summer temperature profile, $T=30 \mathrm{e}^{-0.05 x}$. Results are qualitatively the same for most temperature functions we considered, including those with thermal stratification. We take $\boldsymbol{\ell}$, the phenotypic trait determining competitive ability, to be shell length, assuming that mussels must compete with immediate neighbors for access to the water column. Shell growth is related to temperature through a logistic regression: $\ell(T, t)=\exp \left[a+b_{1} T+b_{2} T^{2}+b_{3} \ell(t-1)\right]-1$, where $\ell$ is shell length, $T$ is temperature in degrees centigrade, and $t$ is time - here taken to be years but in the source study it is a 3month mesocosm experiment (Table 1) (Thorp et al. 1998). Together with our model, this assumes that body growth occurs primarily during a 3 -month summer season. Shell size is related to egg production by $0.4 \cdot(\ell)^{4.4}$ (Walz 1978; Stoeckel et al. 2004), which we assume is the same for both species. We assume that $0.1 \%$ of larvae survive to settle on the lake bottom, similar to other models (Strayer and Malcom 2006) and consistent with other observations (Sprung 1989). Fecundity is then $f(x)=0.001 \cdot 0.4 \cdot[\ell(x)]^{4.4}$, which assumes an excess of sperm. Basal survival rates are also related to temperature by $s_{j, 1}(T)=s_{j, 2}(T)=\left[\exp \left(a+b_{1} T+\right.\right.$ $\left.\left.b_{2} T^{2}\right)\right]\left[\exp \left(a+b_{1} T+b_{2} T^{2}\right)\right]^{-1}$ based on the same mesocosm experiments (Table 1) (Thorp et al. 1998) plus our simplifying assumption that $s_{j, 1}(T)=s_{j, 2}(T)$. 
Table 1 Parameter values of Dreissena survival and shell growth models (Thorp et al. 1998)

\begin{tabular}{llllll}
\hline Model & Species & $a$ & $b_{1}$ & $b_{2}$ & $b_{3}$ \\
\hline \multirow{2}{*}{ Shell growth } & Quagga & -1.39 & 0.49 & -0.0095 & -0.058 \\
& Zebra & -1.39 & 0.34 & -0.015 & -0.058 \\
\multirow{3}{*}{ Survival } & Quagga & -10.81 & 1.046 & -0.023 & - \\
& Zebra & -20.46 & 1.88 & -0.039 & - \\
\hline
\end{tabular}

\section{Appendix 3}

Here, we calculate the expected lifespan of an adult mussel invading a resident community. Let the annual survivorship of an adult be $p$. Let $\tau$ be a random variable for the duration of an adult lifespan. The probability of dying after 1 year is $P[\tau=1]=(1-p)$. The probability of dying after 2 years is $P[\tau=2]=p(1-p)$ and after 3 years is $P[\tau=3]=p^{2}(1-p)$. The probability of dying after $n$ years is $P[\tau=n]=p^{n-1}(1-p)$. The mean duration of an adult is then given by the expectation

$$
\begin{aligned}
E[\tau] & =\sum_{t} t P[\tau=t] \\
& =(1-p)\left(1+2 p+3 p^{2}+4 p^{3}+\ldots\right) \\
& =(1-p) \frac{\mathrm{d}}{\mathrm{d} p}\left[(1-p)^{-1}\right] \\
& =(1-p)^{-1}
\end{aligned}
$$

\section{Appendix 4}

Here we give the next generation operators for Eqs. (30) and (31). Equation (30) has next generation operator

$$
\begin{aligned}
\Gamma n(x, t) & =\int f(0, z) s(0, z)\left(n_{0}(z, t)+n_{1}(z, t)+\mathrm{K}\right) k_{\mathrm{J}}(z, x) \mathrm{dz} \\
n_{0}(x, t) & =n(x, t) \\
n_{i}(x, t) & =\int s(0, z) n_{i-1}(z, t) k_{\mathrm{A}}(z, x) \mathrm{dz}, \quad \mathrm{i}=1,2, \mathbf{K}
\end{aligned}
$$

and Eq. (31) has next generation operator

$$
\begin{aligned}
\Gamma n(x, t) & =\sum_{z} \beta(z) P_{\mathrm{J}}(z)\left(n_{0}(z, t)+n_{1}(z, t)+\mathrm{K}\right) k^{\prime}(z, x) \\
n_{0}(x, t) & =n(x, t) \\
n_{i}(x, t) & =\sum_{z} P_{\mathrm{A}}(z) n_{i-1}(z, t) k^{\prime}(z, x), \quad i=1,2, \mathbf{K}
\end{aligned}
$$

The global net reproductive value $\bar{R}_{0}$ is the spectral radius of $\Gamma$. The fundamental niche and source-sink $R_{0} \mathrm{~s}$ can be calculated using the methods of Appendix 1.

\section{References}

Abrams PA, Wilson WG (2004) Coexistence of competitors in metacommunities due to spatial variation in resource growth rates; does $\mathrm{R}^{*}$ predict the outcome of competition? Ecol Lett 7:929-940. doi:10.1111/j.1461-0248.2004.00644.x
Amarasekare P (2003) Competitive coexistence in spatially structured environments: a synthesis. Ecol Lett 6:1109-1122. doi:10.1046/ j.1461-0248.2003.00530.x

Amarasekare P, Nisbet RM (2001) Spatial heterogeneity, source-sink dynamics, and the local coexistence of competing species. Am Nat 158:572-584. doi:10.1086/323586

Anderson RM, May RM (1991) Infectious diseases of humans. Oxford University Press, Oxford

Boldin B (2006) Introducing a population into a steady community: the critical case, the center manifold, and the direction of bifurcation. SIAM J Appl Math 66:1424-1453. doi:10.1137/050629082

Bridgeman TB, Fahnenstiel GL, Lang GA, Nalepa TF (1995) Zooplankton grazing during the zebra mussel (Dreissena polymorpha) colonization of Saginaw Bay, Lake Huron. J Great Lakes Res 21:567-573

Brown JH, Kodric-Brown A (1977) Turnover rates in insular biogeography - effect of immigration on extinction. Ecology 58:445-449. doi:10.2307/1935620

Case TJ, Holt RD, McPeek MA, Keitt TH (2005) The community context of species' borders: ecological and evolutionary perspectives. Oikos 108:28-46. doi:10.1111/j.0030-1299.2005.13148.x

Caswell H (2001) Matrix population models. Sinauer, Sunderland

Claxton WT, Mackie GL (1998) Seasonal and depth variations in gametogenesis and spawning of Dreissena polymorpha and Dreissena bugensis in eastern Lake Erie. Can J Zool 76:2010 2019. doi:10.1139/cjz-76-11-2010

Cushing J, Zhou Y (1994) The net reproductive value and stability in matrix population models. Nat Resour Model 8:279-333

de Camino-Beck T, Lewis MA (2007) A new method for calculating net reproductive rate from graph reduction with applications to the control of invasive species. Bull Math Biol 69:1341-1354. doi:10.1007/s11538-006-9162-0

Diekmann O, Heesterbeek JAP (2000) Mathematical epidemiology of infectious diseases: model building analysis and interpretation. Wiley, New York

Diekmann O, Heesterbeek JAP, Metz JAJ (1990) On the definition and the computation of the basic reproduction ratio R0 in models for infectious diseases in heterogeneous populations. J Math Biol 28:365-382. doi:10.1007/BF00178324

Fahnenstiel GL, Bridgeman TB, Lang GA, McCormick MJ, Nalepa TF (1995) Phytoplankton productivity in Saginaw Bay, Lake Huron: effects of zebra mussel (Dreissena polymorpha) colonization. J Great Lakes Res 21:465-475

Figueira WF, Crowder LB (2006) Defining patch contribution in source-sink metapopulations: the importance of including dispersal and its relevance to marine systems. Popul Ecol 48:215224. doi:10.1007/s10144-006-0265-0

Fox JW (2007) Testing the mechanisms by which source-sink dynamics alter competitive outcomes in a model system. Am Nat 170:396-408. doi:10.1086/519855

Griffiths RW, Schloesser DW, Leach JH, Kovalak WP (1991) Distribution and dispersal of the zebra mussel (Dreissena polymorpha) in the Great Lakes region. Can J Fish Aquat Sci 48:1381-1388. doi:10.1139/f91-165

Hanski I (1998) Metapopulation dynamics. Nature 396:41-49. doi:10.1038/23876

Hanski I, Gyllenberg M (1993) 2 General metapopulation models and the core-satellite species hypothesis. Am Nat 142:17-41. doi: $10.1086 / 285527$

Hebert PDN, Muncaster BW, Mackie GL (1989) Ecological and genetic studies on Dreissena Polymorpha (Pallas) - a new mollusk in the Great-Lakes. Can J Fish Aquat Sci 46:1587-1591

Heesterbeek JAP (2002) A brief history of $R_{0}$ and a recipe for its calculation. Acta Biotheor 50:189-204. doi:10.1023/A:1016599411804

Holt RD (2003) On the evolutionary ecology of species' ranges. Evol Ecol Res 5:159-178 
Hutchinson GE (1957) Concluding remarks. Cold Spring Harb Symp Quant Biol 22:415-427

Idrisi N, Mills EL, Rudstam LG, Stewart DJ (2001) Impact of zebra mussels (Dreissena polymorpha) on the pelagic lower trophic levels of Oneida Lake, New York. Can J Fish Aquat Sci 58:1430-1441. doi:10.1139/cjfas-58-7-1430

Johnson LE, Carlton JT (1996) Post establishment spread in large scale invasions: dispersal mechanisms of the zebra mussel Dreissena polymorpha. Ecology 77:1686-1690. doi:10.2307/ 2265774

Kawecki TJ (1995) Demography of source-sink populations and the evolution of ecological niches. Evol Ecol 9:38-44. doi:10.1007/ BF01237695

Kot M (2001) Elements of mathematical ecology. Cambridge University Press, Cambridge

Krasnosel'skii MA, Zabreiko PP (1984) Geometrical methods of nonlinear analysis. Springer, Berlin

Levin SA (1974) Dispersion and population interactions. Am Nat 108:207-228. doi:10.1086/282900

Li CK, Schneider H (2002) Applications of Perron-Frobenius theory to population dynamics. J Math Biol 44:450-462. doi:10.1007/ s002850100132

Lubchenco J, Palumbi SR, Gaines SD, Andelman S (2003) Plugging a hole in the ocean: the emerging science of marine reserves. Ecol Appl 13:S3-S7. doi:10.1890/1051-0761(2003)013[0003: PAHITO]2.0.CO;2

Lutscher F, Lewis MA (2004) Spatially-explicit matrix models. J Math Biol 48:293-324. doi:10.1007/s00285-003-0234-6

MacArthur RH (1972) Geographical Ecology. Princeton University Press, Princeton

Mackie GL, Schloesser DW (1996) Comparative biology of zebra mussels in Europe and North America: an overview. Am Zool 36:244-258

May B, Marsden JE (1992) Genetic identification and implications of another invasive species of dreissenid mussel in the Great Lakes. Can J Fish Aquat Sci 49:1501-1506. doi:10.1139/f92-166

Metz JAJ, Nisbet RM, Geritz SAH (1992) How should we define fitness for general ecological scenarios? Trends Ecol Evol 7:198202

Mills EL, Chrisman JR, Baldwin B, Owens RW, O'Gorman R, Howell T, Roseman EF, Raths MK (1999) Changes in the dreissenid community in the lower Great Lakes with emphasis on southern Lake Ontario. J Great Lakes Res 25:187-197

Mills EL, Dermott RM, Roseman EF, Dustin D, Mellina E, Conn DB, Spidle AP (1993) Colonization, ecology, and population structure of the quagga mussel (Bivalvia, Dreissenidae) in the lower Great Lakes. Can J Fish Aquat Sci 50:2305-2314. doi:10.1139/f93-255

Mills EL, Rosenberg G, Spidle AP, Ludyanskiy M, Pligin Y, May B (1996) A review of the biology and ecology of the quagga mussel (Dreissena bugensis), a second species of freshwater dreissenid introduced to North America. Am Zool 36:271-286

Mylius SD, Diekmann O (1995) On evolutionarily stable life histories, optimization and the need to be specific about density dependence. Oikos 74:218-224. doi:10.2307/3545651

Neubert MG (2003) Marine reserves and optimal harvesting. Ecol Lett 6:843-849. doi:10.1046/j.1461-0248.2003.00493.x

Press W, Teukolsky S, Vetterling W, Flannery B (2002) Numerical recipes in $\mathrm{C}++$ : the art of scientific computing, 2nd edn. Cambridge University Press, Cambridge

Pulliam HR (1988) Sources, sinks, and population regulation. Am Nat 132:652-661. doi:10.1086/284880

Pulliam HR (2000) On the relationship between niche and distribution. Ecol Lett 3:349-361. doi:10.1046/j.1461-0248.2000.00143.x
Rex MA, McClain CR, Johnson NA, Etter RJ, Allen JA, Bouchet P, Waren A (2005) A source-sink hypothesis for abyssal biodiversity. Am Nat 165:163-178. doi:10.1086/427226

Roe SL, MacIsaac HJ (1997) Deepwater population structure and reproductive state of quagga mussels (Dreissena bugensis) in Lake Erie. Can J Fish Aquat Sci 54:2428-2433. doi:10.1139/ cjfas-54-10-2428

Runge JP, Runge MC, Nichols JD (2006) The role of local populations within a landscape context: defining and classifying sources and sinks. Am Nat 167:925-938. doi:10.1086/503531

Schreiber SJ, Kelton M (2005) Sink habitats can alter ecological outcomes for competing species. J Anim Ecol 74:995-1004. doi:10.1111/j.1365-2656.2005.00996.x

Smith H, Theime H (2001) Stable coexistence and bi-stability for competitive systems on ordered Banach spaces. J Differ Eqns 176:195-212. doi:10.1006/jdeq.2001.3981

Snyder RE, Chesson P (2004) How the spatial scales of dispersal, competition, and environmental heterogeneity interact to affect coexistence. Am Nat 164:633-650. doi:10.1086/424969

Spidle AP, Mills EL, May B (1995) Limits to tolerance of temperature and salinity in the quagga mussel (Dreissena bugensis) and the zebra mussel (Dreissena polymorpha). Can J Fish Aquat Sci 52:2108-2119. doi:10.1139/f95-804

Sprung M (1989) Field and laboratory observations of Dreissena polymorpha larvae: abundance, growth, mortality and food demands. Arch Hydrobiol 115:537-561

Stoeckel JA, Padilla DK, Schneider DW, Rehmann CR (2004) Laboratory culture of Dreissena polymorpha larvae: spawning success, adult fecundity, and larval mortality patterns. Can J Zool 82:1436-1443. doi:10.1139/z04-125

Stoeckmann A (2003) Physiological energetics of Lake Erie dreissenid mussels: a basis for the displacement of Dreissena polymorpha by Dreissena bugensis. Can J Fish Aquat Sci 60:126-134. doi:10.1139/f03-005

Strayer DL, Malcom HM (2006) Long-term demography of a zebra mussel (Dreissena polymorpha) population. Freshw Biol 51:117130. doi:10.1111/j.1365-2427.2005.01482.x

Thorp JH, Alexander JE, Bukaveckas BL, Cobbs GA, Bresko KL (1998) Responses of Ohio River and Lake Erie dreissenid molluses to changes in temperature and turbidity. Can J Fish Aquat Sci 55:220-229. doi:10.1139/cjfas-55-1-220

Tilman D (1982) Resource competition and community structure. Princeton University Press, Princeton

van Kirk RW, Lewis MA (1999) Edge permeability and population persistence in isolated habitat patches. Nat Resour Model 12:3764

Walz N (1978) The energy balance of the freshwater mussel Dreissena polymorpha Pallas in laboratory experiments and in Lake Constance II. Reproduction. Arch Hydrobiol/Suppl 55:106-119

Wilson AB, Naish KA, Boulding EG (1999) Multiple dispersal strategies of the invasive quagga mussel (Dreissena bugensis) as revealed by microsatellite analysis. Can J Fish Aquat Sci 56:2248-2261. doi:10.1139/cjfas-56-12-2248

Wilson KA, Howell ET, Jackson DA (2006) Replacement of zebra mussels by quagga mussels in the Canadian nearshore of Lake Ontario: the importance of substrate, round goby abundance, and upwelling frequency. J Great Lakes Res 32:11-28. doi:10.3394/ 0380-1330(2006)32[11:ROZMBQ]2.0.CO;2

Wilson WG, Nisbet RM (1997) Cooperation and competition along smooth environmental gradients. Ecology 78:2004-2017

Wilson WG, Nisbet RM, Ross AH, Robles C, Desharnais R (1996) Abrupt population changes along slowly varying environmental gradients. Bull Math Biol 58:907-922 